\title{
Cell layer-specific distribution of transiently expressed barley ESCRT-III component HvVPS60 in developing barley endosperm
}

\author{
Julia Hilscher $^{1} \cdot$ Eszter Kapusi $^{1} \cdot$ Eva Stoger $^{1} \cdot$ Verena Ibl $^{1}$
}

Received: 28 October 2014 / Accepted: 9 March 2015 /Published online: 22 March 2015

(C) The Author(s) 2015. This article is published with open access at Springerlink.com

\begin{abstract}
The significance of the endosomal sorting complexes required for transport (ESCRT)-III in cereal endosperm has been shown by the identification of the recessive mutant supernumerary aleurone layer1 (SAL1) in maize. ESCRT-III is indispensable in the final membrane fission step during biogenesis of multivesicular bodies (MVBs), responsible for protein sorting to vacuoles and to the cell surface. Here, we annotated barley ESCRT-III members in the (model) crop Hordeum vulgare and show that all identified members are expressed in developing barley endosperm. We used fluorescently tagged core ESCRT-III members HvSNF7a/CHMP4 and HvVPS24/CHMP3 and the associated ESCRT-III component HvVPS60a/CHMP5 for transient localization studies in barley endosperm. In vivo confocal microscopic analyses show that the localization of recombinantly expressed HvSNF7a, HvVPS24 and HvVPS60a differs within barley endosperm. Whereas HvSNF7a induces large agglomerations, HvVPS24 shows mainly cytosolic localization in aleurone and subaleurone. In contrast, HvVPS60a localizes strongly at the plasma membrane in aleurone. In subaleurone, HvVPS60a was found to a lesser extent at the plasma membrane and at vacuolar membranes. These results indicate that the steady-state association of ESCRT-III may be influenced
\end{abstract}

Handling Editor: Burkhard Becker

Electronic supplementary material The online version of this article (doi:10.1007/s00709-015-0798-1) contains supplementary material, which is available to authorized users.

Verena Ibl

verena.ibl@boku.ac.at

1 Department of Applied Genetics and Cell Biology, Division of Molecular Cell Biology and Glycobiotechnology, University of Natural Resources and Life Sciences, Muthgasse 18, 1190 Vienna, Austria by cell layer-specific protein deposition or trafficking and remodelling of the endomembrane system in endosperm. We show that sorting of an artificially mono-ubiquitinated Arabidopsis plasma membrane protein is inhibited by HvVPS60a in aleurone. The involvement of HvVPS60a in different cell layer-specific trafficking pathways, reflected by localization of HvVPS60a at the plasma membrane in aleurone and at the PSV membrane in subaleurone, is discussed.

Keywords ESCRT-III · Barley · Endosperm · VPS60 · Cell layer-specific $\cdot$ Seed

\section{Introduction}

In cereals, the endosperm supports the germinating seedling by providing stored nitrogen, carbon and minerals. The fertilized triploid central cell grows into a storage organ where endosperm transfer cells, embryo surrounding tissue cells, aleurone and starchy endosperm (SE) cells carry out specialized functions at different stages of seed development. SE cells accumulate starch and storage proteins (SP) which are mobilized during seed germination via the release of hydrolytic enzymes by the aleurone layer (Olsen 2004). Both SE cells and aleurone cells contain protein storage vacuoles (PSVs). Together with albumins and globulins, prolamins form the major protein component of most cereal grains and are deposited mainly in subaleurone cells (Galili 2004; Shewry et al. 1995). Seed storage proteins (SSPs) reach their final destination by two main routes: soluble albumins and globulins travel through ER and Golgi to PSVs, whereas most prolamins accumulate in ER-derived protein bodies (PBs). Post-Golgi transport of storage proteins towards the vacuole involves dense vesicles and multivesicular bodies (MVBs) (Ibl and Stoger 2012). SSP transport routes depend on the cereal 
species, endosperm tissue layer and developmental timepoint (Ibl and Stoger 2012; Zheng and Wang 2014), and some PBs are ultimately deposited in the PSV after bypassing the Golgi. The massive and complex SSP transport in the endosperm is accompanied by extensive reorganization of the endomembrane system during development (Hoh et al. 1995; Ibl and Stoger 2012; Wang et al. 2010).

In maize, Supernumerary aleurone layer1 (Sal1) was found to restrict aleurone cell identity to the outer cell layer of endosperm (Shen et al. 2003). Sall encodes the maize homolog of endosomal sorting complexes required for transport (ESCRT)-III component Vacuolar Protein Sorting46/Charged Multivesicular Body Protein1 (VPS46/ CHMP1). It is thought that SAL1 maintains the proper plasma membrane concentration of DEFECTIVE KERNEL1 (DEK1) and CRINKLY4 (CR4), both involved in aleurone cell fate specification, by internalization and degradation of SAL1-positive endosomes (Tian et al. 2007). A vps22 (ESCRT-II) mutant in rice endosperm showed seedling lethality and severe reduction in shoot and root growth correlating with the formation of a chalky endosperm. Thus, OsVps22 is supposed to be required for seedling viability and grain filling in rice (Zhang et al. 2013). This is in agreement with electronic northern analyses revealing that the expression levels of most of the ESCRT genes were highest in seed-specific tissues (seed coat and endosperm) of Arabidopsis (Richardson and Mullen 2011). ESCRT originally refers to a protein-protein interaction network in yeast and metazoan cells that coordinates sorting of ubiquitinated membrane proteins into intraluminal vesicles (ILVs) of the MVB (Babst et al. 2002a; Babst et al. 2002b; Katzmann et al. 2001). MVBs then either fuse with lysosomes/ vacuoles leading to degradation of lipids and protein content of ILVs in the vacuolar lumen or they fuse with the plasma membrane, discharging ILVs as exosomes. ESCR T-0, I and II function early in the pathway and are sequentially recruited to endosomes as preformed, stable heteromeric complexes, thereby collecting and concentrating ubiquitinated membrane proteins (Hurley and Emr 2006; Teis et al. 2010; Williams and Urbe 2007). ESCRTIII is necessary for membrane remodelling that drives the biogenesis of MVBs and is further involved in budding of enveloped viruses, abscission of the plasma membrane during cytokinesis, plasma membrane repair (all requiring a topologically similar membrane fission event for budding away from the cytoplasm), surveillance of nuclear pore complex assembly and autophagy (Boura et al. 2012; Buchkovich et al. 2013; Caballe and Martin-Serrano 2011; Carlton and Martin-Serrano 2009; Henne et al. 2013; Hurley and Hanson 2010; Jimenez et al. 2014; Peel et al. 2011; Roxrud et al. 2010; Rusten and Stenmark 2009; Webster et al. 2014). Notably, ESCRT-III is also present in Archaea which lack an endomembrane system, supporting the functional importance of ESCRT-III together with its associated proteins for central aspects of budding processes (Ettema and Bernander 2009; Lindas et al. 2008; Samson and Bell 2009). Whereas ESCRT-III subunits are inactive monomers in the cytoplasm, at membranes, they assemble in a highly ordered manner to generate the transient 450 kDa ESCRT-III complex (Babst et al. 2002a; Teis et al. 2008). The ESCRT-III core complex consists of four subunits which are arranged in two distinct subcomplexes, the Vps20-Snf7 and the Vps2-Vps24 subcomplexes (Babst et al. 2002a). VPS60 and VPS46 are associated proteins and implicated in modulating VPS4 ATPase activity that regulates ESCRT-III disassembly (Hanson and Cashikar 2012). In plants, a cross-species comparison analysis showed for the first time that most ESCRT proteins are present in Arabidopsis thaliana and rice (Winter and Hauser 2006). Since then, intensive in planta research has been conducted to explore the identity, and the structural and functional characteristics of ESCRT proteins in various tissues of rice, maize and Arabidopsis (Cai et al. 2014; Ibl et al. 2012; Katsiarimpa et al. 2011; Katsiarimpa et al. 2013; Katsiarimpa et al. 2014; Korbei et al. 2013; MoulinierAnzola et al. 2014; Reyes et al. 2014; Richardson et al. 2011; Richardson and Mullen 2011; Scheuring et al. 2012; Shahriari et al. 2011; Spallek et al. 2013; Spitzer et al. 2009; Spitzer et al. 2006; Zhang et al. 2013).

The fact that ESCRT-III acts in ILV sorting on MVBswhich represent central hubs in protein sorting to the vacuole and the cell surface - together with the mutant phenotype of sal1 in seed development prompted us to speculate that ESCRT-III may have a pivotal role for cellular homeostasis of protein trafficking and endomembrane dynamics in cereal endosperm. We therefore set out to transfer knowledge from other plant species to the emerging model crop Hordeum vulgare (Saisho and Takeda 2011) with a focus on endosperm, a specialized storage organ and widely used platform for recombinant protein production (Boothe et al. 2010; Magnusdottir et al. 2013; Rademacher et al. 2009; Stoger et al. 2005). We have annotated ESCRT-III members in barley, wheat, rice and maize, and we show that all identified members are expressed during barley endosperm development. Using fluorescently tagged core ESCRT-III components HvSNF7a/CHMP4 and HvVPS24/CHMP3 and the associated ESCRT-III component HvVPS60a/ CHMP5 for transient overexpression and localization studies in barley endosperm, we showed by in vivo confocal microscopy localization in the cytosol, at small agglomerations or at the plasma membrane, respectively. Notably, the subcellular localization of HvVPS60a differs between aleurone and subaleurone and the here shown effect of HvVPS60a on protein sorting of an MVB cargo reporter in aleurone may reflect the involvement of HvVPS60a in diverse trafficking pathways depending on the cell layer. 


\section{Material and methods}

\section{Plant material and growth conditions}

Barley wild-type variety Golden Promise (GP) and its transgenic derivative TIP3-GFP was cultivated as in (Ibl et al. 2014). OsTIP3::TIP3-GFP construct was a gift of Yasushi Kawagoe and is described in (Ibl et al. 2014; Onda et al. 2009).

\section{Database searches for ESCRT-III members in cereals}

Using known protein sequences of ESCRT-III members of A. thaliana (Winter and Hauser 2006), TBLASTN searches for full-length complementary DNA (cDNA) sequences and/ or EST contigs of $H$. vulgare, Triticum aestivum, Zea mays and Oryza sativa Japonica were performed using GenBank at NCBI and following databases: H. vulgare, IPK Barley BLAST Server (http://webblast.ipk-gatersleben.de/barley/); T. aestivum, KOMUGI/Wheat genetic Resources database, Committee for the National BioResource Project Japan (http://www.shigen.nig.ac.jp/wheat/komugi/blast/blast.jsp); Z. mays, POPcorn portal (Cannon et al. 2011) and O. sativa, DFCI Rice Gene Index (http://compbio.dfci.harvard.edu/cgibin/tgi/gimain.pl?gudb=rice). Predicted amino acid sequences were aligned by MUSCLE, and a neighbour joining (NJ) tree (based on aligned sequences with excluding gaps) was constructed to check for consistency of partitioning of proteins to the different ESCRT-III subunits. Both alignment and tree construction were done by programmes implemented in MEGA6 (Tamura et al. 2013). cDNA/EST contig entries coding for proteins with $\mathrm{N}$-terminal or $\mathrm{C}$-terminal deletions or extensions were excluded.

Genomic locations/identifiers for ESCRT-III members which are not annotated in GenBank entries were identified for Z. mays (Maize B73 RefGen_v2), O. sativa and H. vulgare by TBLASTN queries of cDNA to genomic sequences at Ensembl Plants platform or, where unsuccessful for barley and maize, at IPK Barley BLAST Server and POPcorn portal, respectively.

\section{RNA isolation and RT-PCR}

Palea and lemma were removed from 10 to 12 days after pollination (dap) wild-type barley caryopses (Golden Promise) and the embryo cut off. The endosperm was squeezed out of the surrounding pericarp, resulting in sampled endosperm with patches of attached aleurone layer. One hundred milligrams tissue ( $\sim 12-15$ sampled caryopses) was ground in liquid $\mathrm{N}_{2}$, and RNA was isolated following the modified $\mathrm{Li}$ and Trick protocol (Li and Trick 2005) of Mornkham et al. (2013). Two micrograms of RNA was reverse transcribed using First Strand cDNA Synthesis Kit (Thermo Scientific). Gene- specific primers spanned introns derived from alignment of cDNA/EST information against genomic DNA (Table 1, Supplemental Table 1). PCR fragments were separated on $1.5 \%$ agarose gels stained with EtBr. Primers used and expected lengths of fragments are listed in Table S2.

\section{Constructs and molecular biology}

PMA-EGFP (PE) and PMA-EGFP-UB (PEU) reporter constructs were a gift of Swen Schellmann (Herberth et al. 2012).

\section{pActin::VPSx-XFP constructs}

Coding sequences of HvVPS24, HvSNF7a and HvVPS60a were synthesized based on $H$. vulgare Haruna nijo AK366876, AK376941 and AK372130, respectively, and cloned into pBluescript II SK(+) by GeneCust. HvVPS24 and HvSNF7a.1 were synthesized as N-terminal fusions to mCherry (AAV52164.1 (Shaner et al. 2004)) and mEosFP (AAV54099 with V123T and T158H for retaining monomeric state (Wiedenmann et al. 2004)), respectively, and inserting an $X m n I$ restriction site between HvVPSx and XFP sequences. Nucleotide sequences of mCherry and mEosFP were codon optimized for barley using GENEius (Eurofins).

To obtain HvVPS24-mEos driven by the rice actin1 promoter, first, HvVPS24-mCherry was transferred via SalI/ HindIII into the binary vector pSB277 (Kapusi et al. 2013) thereby replacing gfpS65T, followed by exchanging mCherry with mEosFP using restriction sites $X m n \mathrm{I} / A s c \mathrm{I}$. To obtain actin::HvSNF7a-mEosFP, HvVPS24-mCherry was replaced with HvSNF7a-mEosFP using XmnI/AscI. actin::mEosFP was derived from actin::HvSNF7a-mEosFP by deletion of HvSNF7a with SwaI/XmnI. To obtain actin::HvVPS60amCherry, gfpS65T in pSB277 was replaced via SalI/HindIII with HvVPS60a-mCherry.

\section{Transient transformation of barley endosperm cells}

Palea, lemma and dorsal pericarp tissue were removed with forceps from 12 to 15 dap barley caryopses to dissect the aleurone layer. For bombarding subaleurone cells, additionally, the aleurone layer was removed with forceps. Caryopses were placed on one half MS medium supplemented with $1 \%$ sucrose and $3 \mathrm{gl}^{-1}$ phytagel. The Biolistic ${ }^{\circledR}$ PDS-1000/He Particle Delivery System (BIO-RAD) was used for transient transformation. As described by Sanford et al. 1993, $0.6 \mu \mathrm{m}$ gold particles were washed and coated. Bombardment was performed twice with $6 \mu \mathrm{l}$ of coated particles per shot, at 27in. Hg chamber vaccum, 900 psi. Distances from rupture disc to macrocarrier and macrocarrier to stopping screen were approximately 2 and $1 \mathrm{~cm}$, respectively. The target shelf was inserted at position 3 from below. 


\section{Confocal microscopy}

Sections of transiently transformed seeds were mounted in tap water within a small border of vaseline to avoid sample floating, and glue was used to stabilize the cover slips. Images were captured by sequential scan using the Leica SP5 confocal laser scanning microscope with the following filter settings: mEosFP, excitation wavelength $488 \mathrm{~nm}$, emission wavelength 508 $540 \mathrm{~nm}$; for mCherry, excitation 514, emission 580-640 nm; and for GFP, excitation wavelength $488 \mathrm{~nm}$, emission wavelength: 503-530 $\mathrm{nm}$. Images were processed using Leica confocal software version 2.61, ImageJ and Adobe Photoshop CS5.

\section{Statistical analyses}

The mean values of the percentage values were calculated for each biological replicate and the standard deviation (sd) was calculated. The sd should be neglected in cases where only one biological replication exists. We chose a two-sample t-test to calculate the significance as following: t-test was calculated from the percentage values of comparable, different groups that have unequal variances. Data are significant when $P<0.05$.

\section{Results}

\section{Annotation of ESCRT-III members expressed in cereals}

Functional knowledge and the annotation of ESCRT-III components in cereals are missing - with the exception of SAL1 in maize and barley (Olsen et al. 2008; Shen et al. 2003). ESCRT-III members constitute a family of small coiled coil proteins which are divided into the two subfamilies SNF7VPS20-VPS60 and VPS2-VPS24-VPS46; the number of paralogs for each subunit member varies between organisms (Howard et al. 2001; Leung et al. 2008; Winter and Hauser 2006). To build an inventory of ESCRT-III members in cereals, known A. thaliana proteins (Winter and Hauser 2006) were used to search public databases for homologs with evidence for full-length expressed sequences (cDNAs or EST contigs) in barley, wheat, rice and maize. A NJ tree including A. thaliana proteins was used to allocate newly identified members to ESCRT-III subunits (Fig. 1). The corresponding alignment can be found as Supplementary Material 1; a list with database identifiers is provided in Table 1.

Fig. 1 Neighbour joining $(N J)$ tree of 61 protein sequences of ESCRT-III members of $A$. thaliana and barley, wheat, maize and rice presented in Table 1 and based on the alignment provided in Supplemental Figure 1. The NJ tree was built using standard configurations in MEGA6 (Tamura et al. 2013). Distances using the Poisson correction method are given in the units of the number of amino acid substitutions per site. All positions containing gaps were eliminated. Bootstrap values (1000 replicates) are shown at the branches

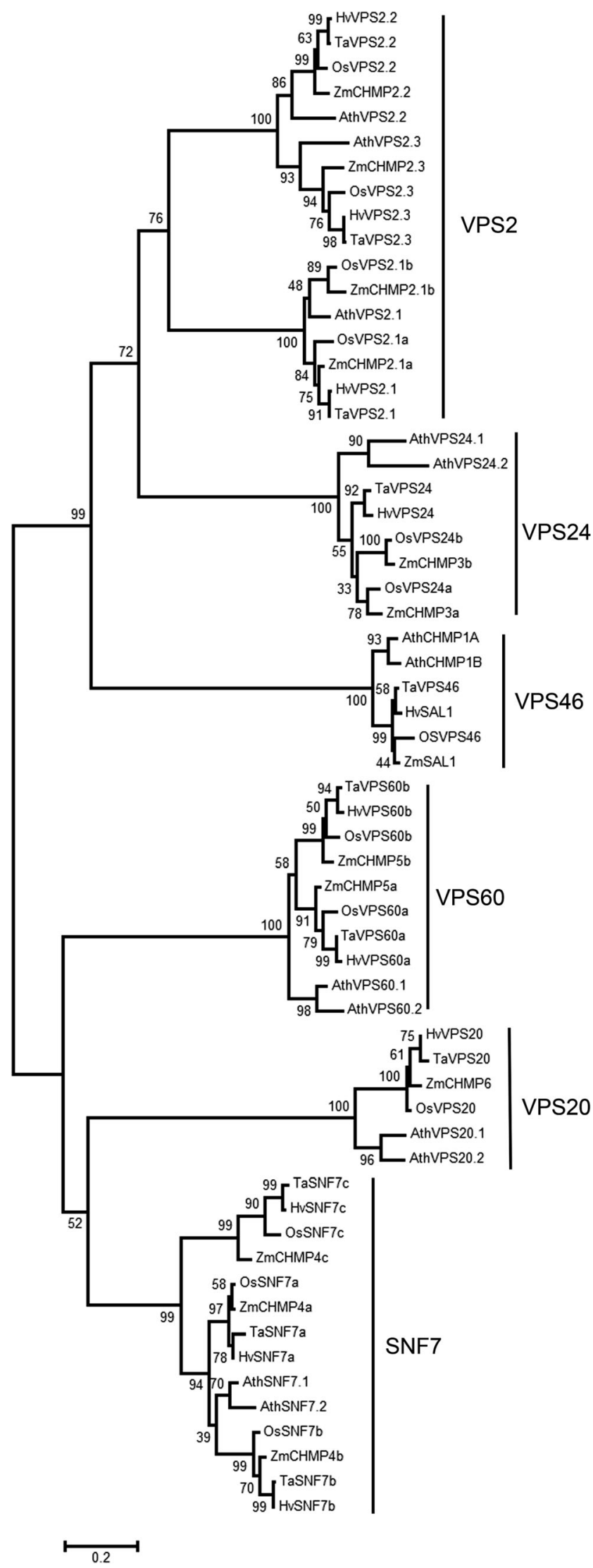


Table 1 Inventory of evidently transcribed ESCRT-III members in Hordeum vulgare, Triticum aestivum, Oryza sativa ssp. japonica and Zea mays

\begin{tabular}{|c|c|c|c|c|c|}
\hline & \multirow[t]{2}{*}{ Name } & \multicolumn{4}{|l|}{ Accession number } \\
\hline & & Full-length cDNA/EST contig & Derived protein & aa & Genomic identifier $^{\mathrm{g}}$ \\
\hline \multicolumn{6}{|l|}{ VPS2/CHMP2 } \\
\hline \multirow[t]{3}{*}{ H. vulgare ${ }^{\mathrm{h}}$} & VPS2.1 & AK250448 & - & 224 & MLOC_67016 \\
\hline & VPS2.2 & AK375733 & BAK06928 & 229 & MLOC_10666 ${ }^{\mathrm{f}}$ \\
\hline & VPS2.3 & AK368215 & BAJ99418 & 212 & MLOC_16800 ${ }^{\mathrm{f}}$ \\
\hline \multirow[t]{3}{*}{ T. aestivum } & VPS2.1 & AK330978 & - & 224 & nd \\
\hline & VPS2.2 & Contig14460 a & - & 229 & nd \\
\hline & VPS2.3 & Contig $17212^{\mathrm{a}}$ & - & 212 & nd \\
\hline \multirow[t]{4}{*}{ O. sativa } & VPS2.1a & NM_001075069 & NP_001068537 & 224 & Os11g0703400 \\
\hline & VPS2.1b & NM_001065783 & NP_001059248 & 225 & Os07g0236800 \\
\hline & VPS2.2 & NM_001057274 & NP_001050739 & 229 & Os03g0639800 \\
\hline & VPS2.3 & NM_001071383 & NP_001064848 & 212 & Os10g0476400 \\
\hline \multirow[t]{4}{*}{ Z. mays } & CHMP2.1a & EU969317 & ACG41435 & 224 & Scaffold $340^{\text {ef }}$ \\
\hline & CHMP2.1b & NM_001139057 & NP_001132529 & 223 & GRMZM2G431900 \\
\hline & CHMP2.2 & NM_001157066 & NP_001150538 & 229 & GRMZM2G046676 \\
\hline & CHMP2.3 & NM_001155163 & NP_001148635 & 212 & GRMZM2G004996 \\
\hline \multirow[t]{3}{*}{ A. thaliana } & VPS2.1 & NM_126650 & NP_565336 & 225 & At2g06530 \\
\hline & VPS2.2 & NM_123823 & NP_199269 & 222 & At5g44560 \\
\hline & VPS2.3 & NM_100276 & NP_563696 & 210 & Atlg03950 \\
\hline \multicolumn{6}{|l|}{ VPS 24/CHMP3 } \\
\hline H. vulgare ${ }^{\mathrm{h}}$ & VPS24 & AK366876 & BAJ98079 & 230 & MLOC_73329 \\
\hline T. aestivum & VPS24 & AK333579 & - & 230 & nd \\
\hline \multirow[t]{2}{*}{ O. sativa } & VPS24a & AK242193 & - & 231 & Os03g01810 \\
\hline & VPS24b & TC516772 ${ }^{\mathrm{b}}$ & - & 229 & Os07g29630 \\
\hline \multirow[t]{2}{*}{ Z. mays } & СНMP3a & EU953837 & ACG25955 & 228 & GRMZM2G042552 \\
\hline & CHMP3b & EU956407 & ACG28525 & 229 & GRMZM2G165195 \\
\hline \multirow[t]{2}{*}{ A. thaliana } & VPS24.1 & NM_122201 & NP_197686 & 229 & At5g 22950 \\
\hline & VPS24.2 & NM_114369 & NP_190086 & 200 & At3g45000 \\
\hline \multicolumn{6}{|l|}{ VPS20/CHMP6 } \\
\hline H. vulgare ${ }^{\mathrm{h}}$ & VPS20 & AK357563 & BAJ88777 & 229 & MLOC_30872 \\
\hline T. aestivum & VPS20 & Contig13879 a & - & 229 & - \\
\hline O. sativa & VPS20 & NM_001053857 & NP_001047322 & 228 & Os02g0596500 \\
\hline Z. mays & CHMP6 & NM_001143085 & NP_001136557 & 229 & GRMZM2G122983 \\
\hline \multirow[t]{2}{*}{ A. thaliana } & VPS20.1 & NM_125783 & NP_568980 & 219 & At5g63880 \\
\hline & VPS20.2 & NM_120962 & NP_196488 & 216 & At5g09260 \\
\hline \multicolumn{6}{|l|}{ SNF7/CHMP4 } \\
\hline \multirow[t]{3}{*}{ H. vulgare ${ }^{\mathrm{h}}$} & SNF7a & AK376941 & BAK08135 & 220 & MLOC_42957 ${ }^{\mathrm{f}}$ \\
\hline & SNF7b & AK354835 & BAJ86054 & 222 & MLOC_16305 ${ }^{\mathrm{f}}$ \\
\hline & SNF7c & AK366149 & BAJ97352 & 218 & MLOC_17778 ${ }^{\mathrm{f}}$ \\
\hline \multirow[t]{3}{*}{ T. aestivum } & SNF7a & AK333051 & - & 220 & nd \\
\hline & SNF7b & AK331201 & - & 222 & nd \\
\hline & SNF7c & Contig9162 a & - & 217 & nd \\
\hline \multirow[t]{3}{*}{ O. sativa } & SNF7a & NM_001069258 & NP_001062723 & 220 & Os09g0267600 \\
\hline & SNF7b & NM_001064575 & NP_001058040 & 220 & Os06g0608500 \\
\hline & SNF7c & NM_001072148 & NP_001065616 & 216 & Os11g0123500 \\
\hline \multirow[t]{3}{*}{ Z. mays } & CHMP4a & NM_001254842 & NP_001241771 & 222 & GRMZM2G107757 \\
\hline & CHMP4b & EU975735 & ACG47853 & 226 & GRMZM2G044805 \\
\hline & CHMP4c & $\mathrm{TC} 482666^{\mathrm{d}}$ & - & 223 & GRMZM2G103217 \\
\hline A. thaliana & SNF7.1/VPS32.2 & NM_119060 & NP_194645 & 219 & At4g29160 \\
\hline & SNF7.2/VPS32.1 & NM_127541 & NP_179573 & 213 & At2g19830 \\
\hline VPS46/CHMP1 & & & & & \\
\hline H. vulgare ${ }^{\mathrm{h}}$ & SAL1 (Tian et al., 2007) & AK252514 & ABW81400 & 204 & MLOC_57384 \\
\hline T. aestivum & VPS46 & AK335766 & - & 204 & nd \\
\hline O. sativa & VPS46 & NM_001064712 & NP_001058177 & 205 & Os06g0643300 \\
\hline
\end{tabular}


Table 1 (continued)

\begin{tabular}{|c|c|c|c|c|c|}
\hline & \multirow[t]{2}{*}{ Name } & \multicolumn{4}{|l|}{ Accession number } \\
\hline & & Full-length cDNA/EST contig & Derived protein & aa & Genomic identifier $^{\mathrm{g}}$ \\
\hline Z. mays & SAL1 (Shen et al., 2003) & NM_001111748 & NP_001105218 & 204 & GRMZM2G117935 \\
\hline \multirow[t]{2}{*}{ A. thaliana } & CHMP1A, VPS46.2 & NM_105961 & NP_565053 & 203 & At1g73030 \\
\hline & CHMP1B, VPS46.1 & NM_101635 & NP_173215 & 203 & At1g17730 \\
\hline \multicolumn{6}{|l|}{ VPS60/CHMP5 } \\
\hline \multirow[t]{2}{*}{ H. vulgare ${ }^{\mathrm{h}}$} & VPS60a & AK372130 & BAK03328 & 232 & MLOC5296 \\
\hline & VPS60b & AK367793 & BAJ98996 & 228 & barke_contig_1823961 ${ }^{\mathrm{f}}$ \\
\hline \multirow[t]{2}{*}{ T. aestivum } & VPS60a & AK332171 & - & 232 & nd \\
\hline & VPS60b & tplb0015n $24^{c}$ & - & 228 & nd \\
\hline \multirow[t]{2}{*}{ O. sativa } & VPS60a & NM_001060924 & NP_001054389 & 232 & Os05g0102900 \\
\hline & VPS60b & NM_001185188 & NP_001172117 & 232 & Os01g0102950 \\
\hline \multirow[t]{2}{*}{ Z. mays } & CHMP5a & NM_001157823 & NP_001151295 & 228 & GRMZM2G092468 \\
\hline & CHMP5b & BT040588 & ACF85593 & 230 & GRMZM2G069827 \\
\hline \multirow[t]{2}{*}{ A. thaliana } & VPS60.1 & NM_111900 & NP_187675 & 235 & At3g10640 \\
\hline & VPS60.2 & NM_120567 & NP_568143 & 235 & At5g04850 \\
\hline
\end{tabular}

Footnotes explain database identifiers other than those of GenBank. Cereal ESCRT-III subgroup member designation was chosen to follow $A$. thaliana nomenclature with numbering for VPS2 in cases of cereal members grouping together with the corresponding A. thaliana member. Alphabetic characters were used for cereal members where this is not the case (i.e. SNF7a, b, c). Arabidopsis members have been annotated in (Winter and Hauser 2006)

${ }^{\text {a }}$ EST contig identifier of KOMUGI/Wheat genetic Resources database database

b DFCI Oryza sativa Gene Index (OsGI): http://compbio.dfci.harvard.edu/cgi-bin/tgi/tc_report.pl?tc=TC516772\&species=rice

c cDNA identifier of KOMUGI/Wheat genetic Resources database database

${ }^{\text {d }}$ DFCI Zea mays Gene Index (ZmGI): http://compbio.dfci.harvard.edu/cgi-bin/tgi/tc_report.pl?species=maize\&tc=TC482666

e B73 RefGen_v3 Scaffold 340, incomplete

${ }^{\mathrm{f}}$ Genomic identifier does not cover full length of transcript

g Genomic identifiers of barley are based on Hordeum vulgare Morex genome

${ }^{\mathrm{h}}$ cDNA/protein sequences are from Hordeum vulgare Haruna Nijo

${ }^{i} 5^{\prime}$ region of cDNA is not aligned to genomic identifier

Components with evidence for expression were detected for all ESCRT-III subunits in barley, wheat, maize and rice (Table 1, Fig. 1). In each of these species, one homolog was detected for VPS2.2, VPS2.3, VPS20 and VPS46. Two and three homologs were identified for VPS60 and SNF7, respectively. Barley and wheat possess one and maize and rice two homologs of VPS2.1 and VPS24. On the NJ tree, each of the two Arabidopsis paralogs for VPS20, VPS24, VPS46 and VPS60 is found as separate clades to cereal members on respective subtrees. AtVPS2.1, AtVPS2.2 and AtVPS2.3 are placed within monophyletic groups with the respective cereal members. The $A$. thaliana paralogs SNF7.1 and SNF7.2 are placed with high bootstrap support, yet as separate clade, with cereal SNF7a and SNF7b.

\section{Expression analysis of ESCRT-III in barley endosperm}

Mass spectrometry was previously used to construct an atlas of developing maize seed proteotypes comprising approximately 14,000 proteins extracted from embryo, endosperm and aleurone/pericarp fractions at seven stages of development (Walley et al. 2013). We used the atlas to search for ESCRT-
III members. Normalized and averaged data show that members of all ESCRT-III subunits are expressed during development in endosperm and in aleurone/pericarp tissue in maize (Table S2). Based on the Affymetrix Barley1 Gene Chip, expression data of grain maturation, desiccation and germination of two tissue fractions (starchy endosperm/aleurone and embryo/scutellum) are available for barley (Sreenivasulu et al. 2008). They indicate that homologs to Arabidopsis ESCRTIII (HvVPS2.1, HvSAL1, HvSNF7a/c, HvVPS24, $\mathrm{HvVPS60a/b)} \mathrm{are} \mathrm{expressed} \mathrm{during} \mathrm{analysed} \mathrm{stages} \mathrm{of} \mathrm{grain}$ development and germination. Furthermore, homologs to AtVPS28 (ESCRT-I) and AtVPS36 (ESCRT-II) are active (Table S3).

To establish whether all detected ESCRT-III family members are expressed in barley endosperm, real-time PCR (RTPCR) was performed using RNA isolated from endosperm tissue (including starchy endosperm and aleurone) of 12dap-old caryopses. All ESCRT-III members of barley are expressed in endosperm tissue (Fig. 2a, b) supporting a possible functional role of ESCRT in endosperm development, in line with sal1 and vps22 mutants in maize and rice, respectively (Shen et al. 2003; Zhang et al. 2013). 

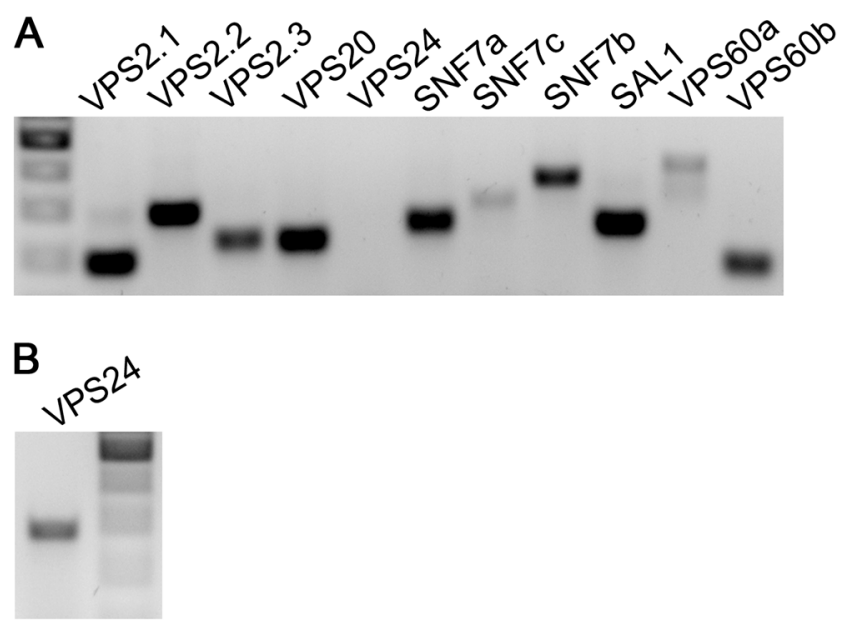

Fig. 2 ESCRT-III members are expressed in barley endosperm. Genespecific primers were used to detect expression of ESCRT-III members in barley endosperm cDNA (10-12 dap). a PCR on 1:10 diluted cDNA of barely endosperm. b PCR for VPS24 on undiluted cDNA of barley endosperm. DNA marker 500-200 bp

\section{ESCRT-III members are distributed in the cytosol, at agglomerations and at the plasma membrane in barley endosperm}

To study the subcellular localization of ESCRT-III, barley ESCRT-III members SNF7a, VPS24 and VPS60a were chosen for transient expression studies by particle bombardment of barley endosperm. The genes were placed under the control of the rice actin 1 promoter (McElroy et al. 1991) and fluorescently tagged at their C-terminus with fluorescent proteins mEos and mCherry, which have been chosen for their ability to retain a monomeric state (see "Materials and methods"). C-terminally tagged plant ESCRT-III members AtVPS2.1 and AtVPS2.2 exhibited dominant negative effects (Ibl et al. 2012; Katsiarimpa et al. 2013) probably because of impaired auto-inhibition as analysed for C-terminally tagged yeast and mammalian ESCRT-III proteins (Teis et al. 2008; Zamborlini et al. 2006). Auto-inhibition is regulated via closed and open conformation of the C-terminal alpha-helix and ensures regulation of assembly and disassembly of the ESCRT-III complex at membranes (Henne et al. 2012). Release of auto-inhibition leads to enhanced accumulation on endosomes, termed class $\mathrm{E}$ compartments in yeast (Teis et al. 2008). The experimental setup therefore allowed us to analyse the distribution and behaviour of ESCRT-III members in cells with superimposed recombinant ESCRT-III load. After $24 \mathrm{~h}$, barley endosperm was screened for expression by in vivo (fluorescence and) confocal microscopy, revealing strong fluorescent signals in barley endosperm. In addition to very weak signals in the cytosol and in the nucleus, a strong signal of HvSNF7a-mEosFP could be detected at agglomerations of different sizes (Fig. 3a). HvVPS24-mEosFP localizes in the cytosol and at agglomerations in aleurone cells, highlighted by a maximal z-projection (Fig. 3b). HvVPS60a-mCh could be visualized strongly at the plasma membrane, at agglomerations and in the cytosol (Fig. 3c). Contrary, a maximal z-projection of actin::mEosFP showed a weak signal in the cytosol and in the nucleus, confirming the authenticity of the signals from the barley ESCRT-III fusion proteins (Suppl. Fig. 1). In summary, confocal microscopy studies showed that the recombinant barley ESCRT-III members HvSNF7a, HvVPS24 and HvVPS60a are localized in the cytosol and at agglomerations and HvVPS60a additionally at the plasma membrane in barley endosperm.

\section{Localization of HvVPS60a at the plasma membrane in aleurone versus association with PSV membranes in subaleurone}

Recent data show the distinct behaviour of the endomembrane system in barley aleurone, subaleurone and starchy endosperm during seed development (Ibl et al. 2014). While the morphology of protein storage vacuoles (PSVs) remains unchanged in aleurone cells, PSVs in subaleurone and starchy endosperm are extremely dynamic, reflecting a constantly remodelling endomembrane system in these layers. To test if the subcellular distribution of ESCRT-III differs between cell layers, HvVPS24-mCh and HvVPS60a-mCh were transiently expressed in OsTIP3::TIP3-GFP lines (Ibl et al. 2014) to specifically study the localization of HvVPS24-mCh and HvVPS60a-mCh in both aleurone and in subaleurone. In aleurone, 18 positively transformed cells (biological replicates 1) show that HvVPS24-mCh localizes in all cells diffusely in the cytoplasm, additionally in $78 \%$ of cells at agglomerations and $22 \%$ at the plasma membrane (Fig. 4a, c). However, the intensity of the plasma membrane signal is not increased compared to the cytosolic signal (Suppl. Fig. 2). In subaleurone, HvVPS24-mCh localizes in 13 cells (biological replicates 3) to $100 \%$ cytosolic and to $62 \%$ at agglomerations, showing that the localization of HvVPS24-mCh is similar in aleurone and subaleurone cells (Fig. 4a). Moreover, HvVPS24-mCh is not associated to PSV membranes (Fig. 4a, subaleurone, double arrows). Contrary, the distribution of HvVPS60a-mCh differs between aleurone and subaleurone (Fig. 4b, d). In aleurone, confocal microscopy studies (biological replicates $1, n=$ 14) revealed a weak signal for HvVPS60a-mCh in the cytosol (100\% of cells), at cytoplasmic agglomerations (86\% of cells) (Fig. 4a, asterisks) and strongly at the plasma membrane (100\% of cells) (Fig. 4b, arrow). To investigate HvVPS60a$\mathrm{mCh}$ localization at the plasma membrane further, we used the Arabidopsis integral plasma membrane ATPase (PMA-EGFP; PE; (Herberth et al. 2012)) as a marker (Suppl. Fig. 3). Confocal single scans of co-bombarded cells reveal colocalization at the plasma membrane (Fig. 4e). In subaleurone, 


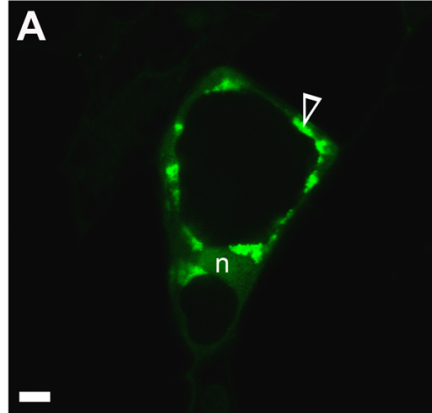

SNF7-mEosFP; $24 \mathrm{~h}$

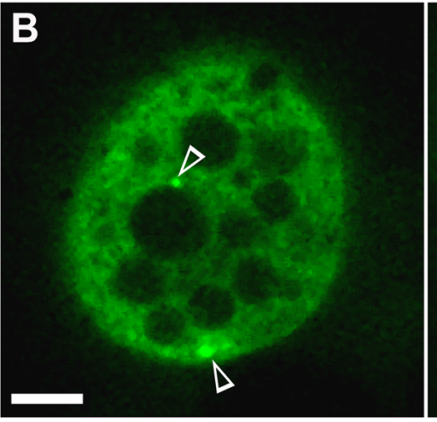

VPS24-mEosFP:

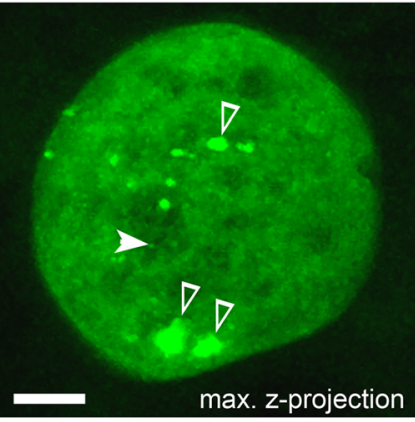

max. z-projection $24 h$

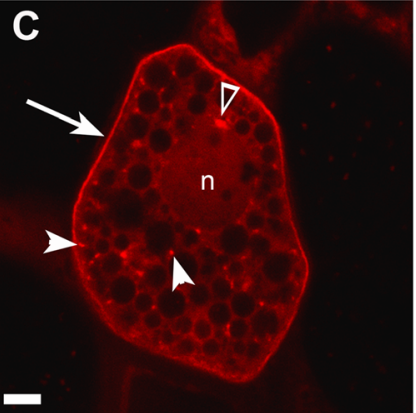

VPS60a-mCh; 24h
Fig. 3 Localization of HvSNF7a, HvVPS24 and HvVPS60a in barley endosperm. a Actin::HvSNF7a-mEosFP induces large agglomerations (open arrowhead) within a cell in the embryo surrounding region. Note the weak signal in the nucleus (n). b Actin::HvVPS24-mEosFP localizes at vesicles (arrowhead) and to the cytoplasm concomitant with inducing

we observed (biological replicates 5, $n=26$ ) a strong cytosolic signal (96\% of cells), fewer agglomerations (62\% of cells) and a reduced number of cells with signal at the plasma membrane (42\% of cells). Moreover, a possible association with TIP3-GFP-labelled PSV membranes could be observed in $50 \%$ of the cells (Fig. 4b, double arrow). In detail, a TIP3-GFP-labelled PSV membrane shows strong labelling of HvVPS60$\mathrm{mCh}$ (Fig. 4f, double arrow), indicating co-localization at the tonoplast. Altogether, these data may indicate a spatially regulated localization pattern of HvVPS60a$\mathrm{mCh}$ in barley aleurone.

\section{Re-localization of HvVPS60a and HvVPS24 to large agglomerations in both aleurone and subaleurone}

As it is known that components of ESCRT complexes can be re-localized as a consequence of their interaction, we subsequently co-transformed the two putative interaction partners (Richardson et al. 2011) HvVPS60a-mCh and VPS24-mEosFP into both barley aleurone and subaleurone. Confocal microscopy analyses of co-transformed cells (aleurone $n=8$; subaleurone $n=4$; biological replicates aleurone/subaleurone 5/1) reveal re-localization of both HvVPS60a-mCh and HvVPS24-mEosFP to large, globular structures (Fig. 5a, b, arrowheads). In detail, previous nearly $100 \%$ cytosolic localization of both HvVPS24 and HvVPS60a in aleurone is reduced to $50 \%$ (Fig. 5c, d), whereas the accumulation of HvVPS2 4 at the plasma membrane increased from $20 \%$ of singly transformed cells to $50 \%$ of co-bombarded cells in aleurone and to approximately $30 \%$ in subaleurone (Fig. 5c). These in vivo microscopic data suggest that the localization of transiently expressed ESCRT-III proteins is influenced by simultaneous overexpression of putative interaction partners. agglomerations (open arrowheads). Note the maximal z-projection of 16 1- $\mu$ m sections. c Actin::HvVPS60a-mCh localizes at the PM (arrow), vesicles (arrowhead), agglomerations (open arrowhead) and to a minor extent in the nucleus $(n)$. Confocal single scans were made $24 \mathrm{~h}$ after bombardment. Scale $=5 \mu \mathrm{m}$

\section{HvVPS60a inhibits vacuolar sorting of a monoubiquitinated plasma membrane protein in aleurone but not in subaleurone}

To investigate whether overexpressed HvVPS24 or HvVPS60a have an inhibitory effect on vacuolar sorting of plasma membrane protein, the endocytosis of an artificial

Fig. 4 The localization of HvVPS60a-mCh differs in aleurone and subaleurone and shows plasma membrane localization in aleurone. a Single scans of barley aleurone and subaleurone cells showing transient transformation of HvVPS24-mCh in TIP3-GFP lines. In both, aleurone and subaleurone, HvVPS24-mCh localizes cytosolic and at small agglomerations (open arrowheads). Note the close ups to visualize colocalization. b Confocal single scans of HvVPS60a-mCh in TIP3-GFP lines and of co-transformation of HvVPS60a-mCh with TIP3-GFP. In aleurone, HvVPS60a-mCh shows strong signal at the pm (arrow), weak in the cytosol in parallel to small agglomeration structures (open arrowheads). Note the altered localization of HvVPS60a-mCh in subaleurone cells. HvVPS60a-mCh signal could be detected close to heterogenous, spherical PSVs (double arrow) but reduced at the PM (arrow). Note the close ups to visualize co-localization. c, d Localization of HvVPS24-mCh and HvVPS60a-mCh in aleurone and subaleurone. Relative number of cells showing localization in cytosol at agglomerations and at the PM was scored. Total number of cells: HvVPS24-mCh in aleurone (biological replicates $1 ; n=18$ ) and in subaleurone (biological replicates $3 ; n=13$ ); HvVPS60a-mCh in aleurone (biological replicates $1 ; n=14$ ) and in subaleurone (biological replicates $3 ; n=24)$. The difference in the localization of HvVPS60a-mCh at the $\mathrm{PM}$ in aleurone and subaleurone is statistically significant $(P<0.05)$. Plus ends of the standard deviation are indicated. e Single scan of an aleurone cell showing co-localization of the plasma membrane marker PMA-EGFP (PE) and HvVPS60a-mCh at the pm (arrow). Fluorescence profile: $X$-axis, distance $[\mu \mathrm{m}]$ from 0 to $6 \mu \mathrm{m} ; Y$ axis, intensity from 0 to 100 . f Single confocal scan of a subaleurone cell that shows co-localization of HvVPS60a-mCh and the vacuolar membrane signal of the TIP3-GFP line. Note the double arrows indicating co-localization of HvVPS60a-mCh with the PSV membrane. Fluorescence profile: $X$-axis, distance $[\mu \mathrm{m}]$ from 0 to $8 \mu \mathrm{m} ; Y$-axis, intensity from 0 to 100 . Confocal single scans and $z$-series were made $24 \mathrm{~h}$ after bombardment. Scale $=5 \mu \mathrm{m}$ 

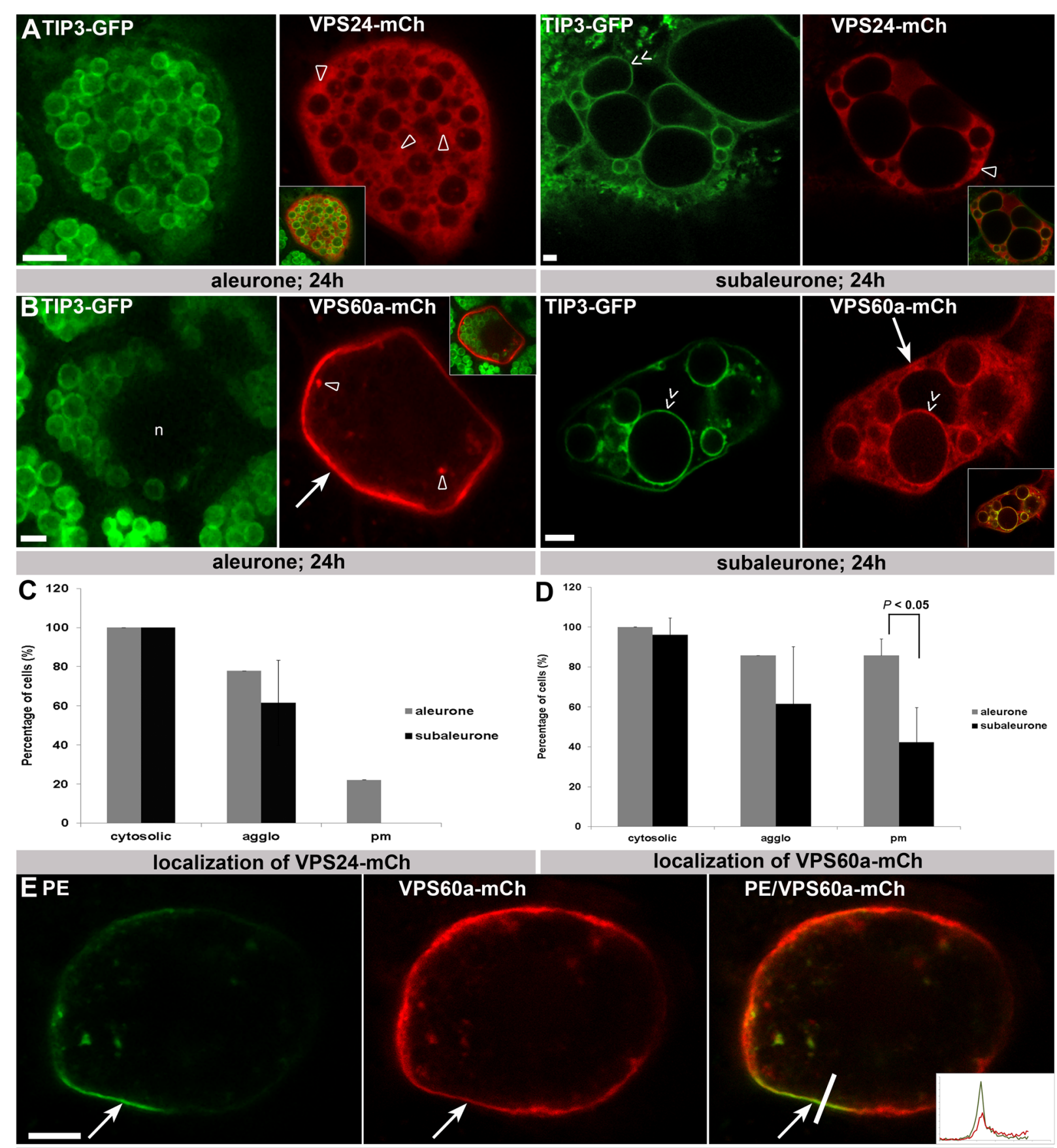

aleurone; $12 \mathrm{~h}$

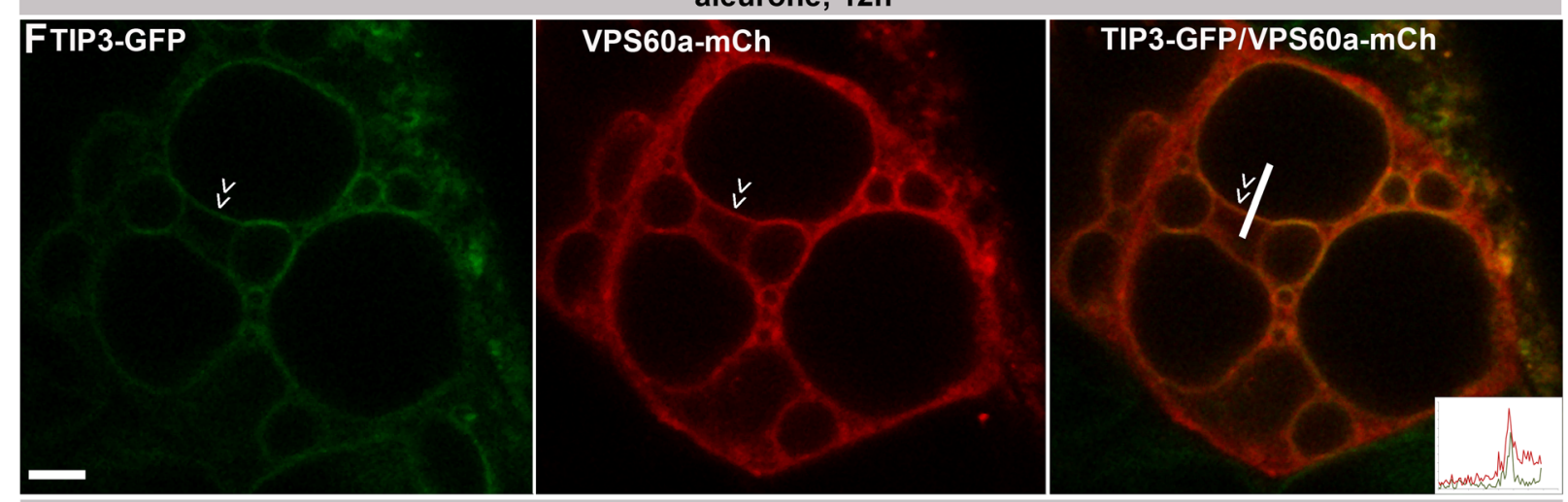

subaleurone; $24 \mathrm{~h}$ 

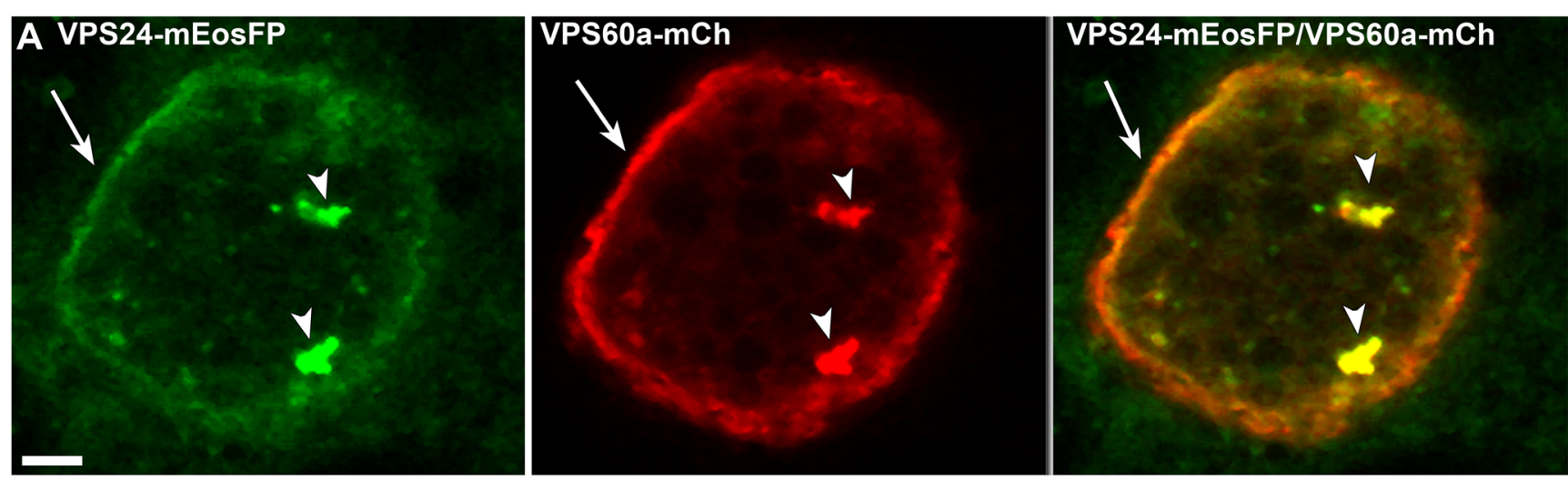

aleurone; $24 \mathrm{~h}$
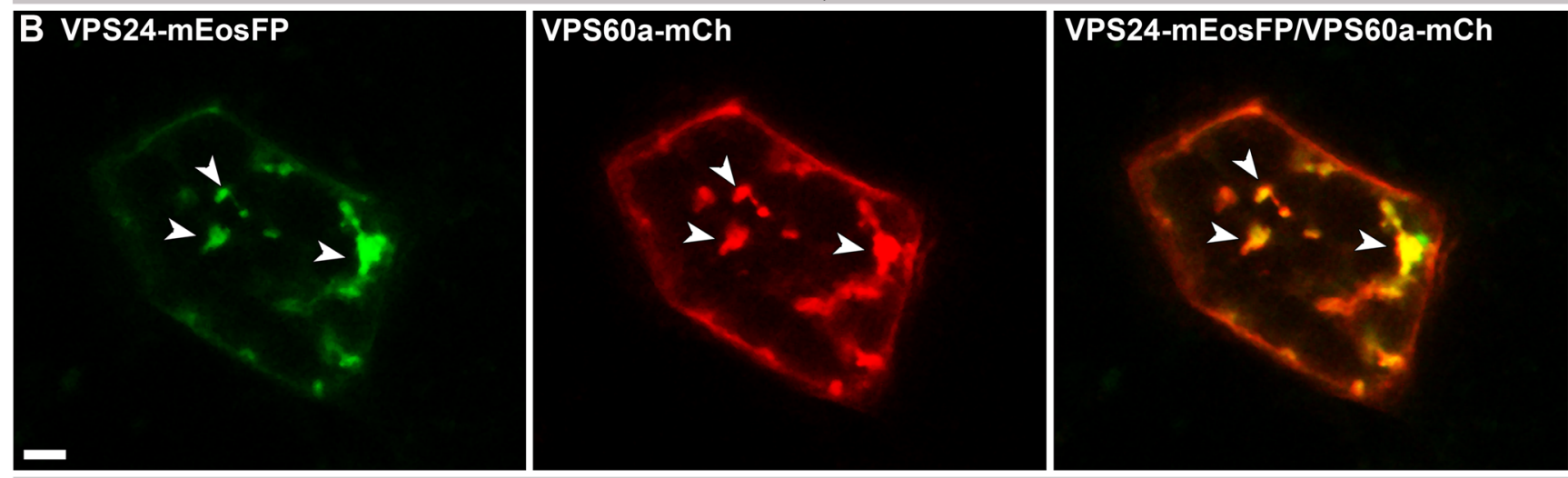

subaleurone; $24 \mathrm{~h}$

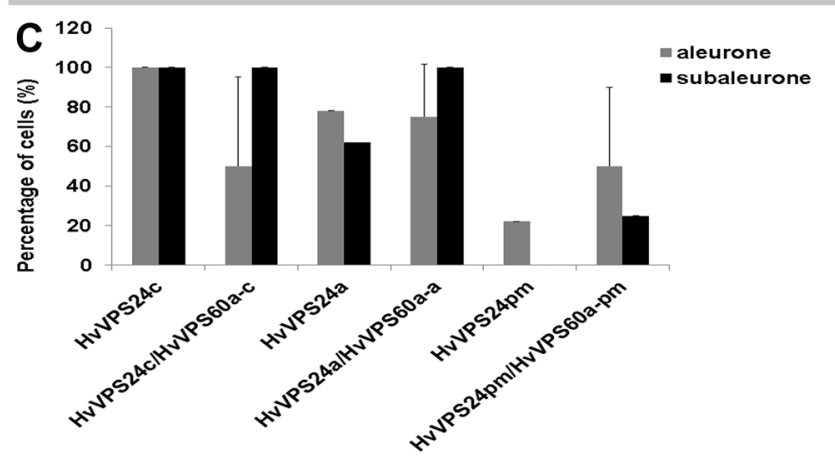

VPS24 co-localization with VPS60a

Fig 5 Co-bombardment of HvVPS24-mEosFP with HvVPS60a-mCh induces re-localization of both proteins to large agglomerations in aleurone and subaleurone. a, b HvVPS24m-Ch and HvVPS60a-mCh are re-localized from cytoplasm to large globulare structures (arrowheads) in aleurone and subaleurone. Note the additional localization of VPS24m-Ch and VPS60a-mCh at the PM in aleurone (arrows). c, d 8 and 4 co-transformed cells within 5 and 1 biological

MVB cargo PMA-EGFP-UB (PEU) was investigated in aleurone and subaleurone cells after co-transformation with HvVPS24m-Ch or HvVPS60a-mCh. Monoubiquitination of the Arabidopsis plasma membrane ATPase PMA-EGFP (PE) induces the endocytosis and sorting into the vacuolar lumen (Herberth et al. 2012). As previously reported, co-expression of both, a dominant negative version of the Arabidopsis AAAATPase AtSKD1(EQ) that is required for the disassembly of ESCRT-III and the ESCRT-III component VPS2.1 fused to a

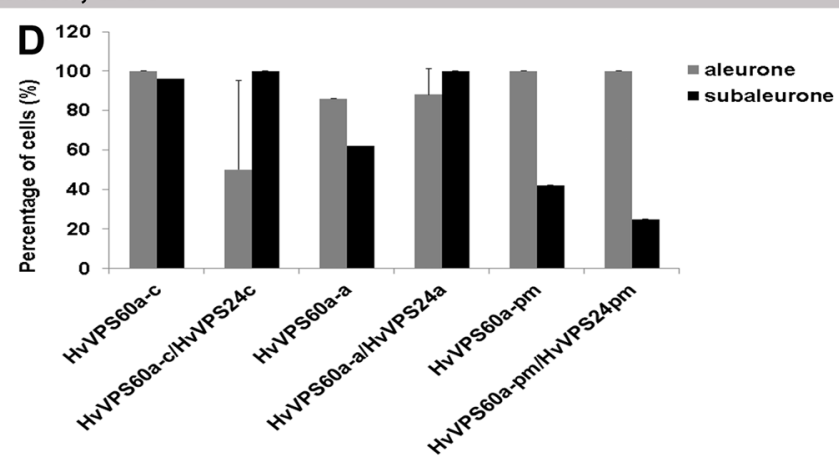

\section{VPS60a co-localization with VPS24}

replicates, respectively, were analysed in aleurone and subaleurone. Relative number of cells showing localization in cytosol $(-c)$, at agglomerations $(-a)$ and at the plasma membrane (-pm) was scored and compared with single transformation. Plus ends of the standard deviation are indicated. Confocal single scans were made $24 \mathrm{~h}$ after bombardment. Scale $=5 \mu \mathrm{m}$.

C-terminal TagRFP, have an inhibitory effect on the sorting of PEU via the MVB pathway towards the vacuole (Herberth et al. 2012; Katsiarimpa et al. 2013). We transiently transformed PE and PEU in aleurone and subaleurone cells and observed PE at the pm and PEU in the vacuolar lumen within $12 \mathrm{~h}$ after transformation (Suppl. Fig. 3). Twelve hours after co-bombardment of PEU with HvVPS24-mCh in aleurone, we could observe PEU at the pm (Fig. 6a, arrow) and in the vacuolar lumen (Fig. 6a, asterisk), similar to the localization of 
PEU alone (Fig. 6a, c). When we analysed PEU in cotransformed HvVPS60a-mCh aleurone cells, PEU was detected at the plasma membrane (Fig. 6a, arrow), at agglomerations (Fig. 6a, open arrowhead), at the vacuolar membrane (Fig. 6a, arrowheads) and approximately in $40 \%$ of the cells in the vacuolar lumen (Fig. 6c). Interestingly, analyses of HvVPS24-mCh and HvVPS60a-mCh in co-bombarded PEU subaleurone cells show localization of PEU at the plasma membrane (Fig. 6b, arrows) and in the vacuolar lumen (Fig. 6b, asterisks). As we could not detect differences in the qualitative fluorescence signal in the vacuolar lumen between PEU single transformation and PEU together with HvVPS60a-mCh, we conclude that HvVPS60a-mCh has hardly any inhibitory effect on the sorting of monoubuquitinated pm ATPase in subaleurone (Fig. 6b arrow, asterisks). Contrary to the PEU signal at the vacuolar membrane in co-bombarded HvVPS60a-mCh aleurone cells, where PEU was only detected at the vacuolar membrane but within the same cell not additionally in the vacuolar lumen, the PEU signal at the vacuolar membrane in co-bombarded HvVPS60a-mCh subaleurone cells could be detected at the vacuolar membrane concomitant with the signal in the vacuolar lumen (Fig. 6d). Thus, the sorting of PEU in HvVPS60a-mCh co-bombarded subaleurone cells is still provided (Fig. 6e). Together, these results indicate that HvVPS60a-mCh is involved in the vacuolar lumen sorting of monoubiquinated plasma membrane cargo in aleurone but not in subaleurone.

\section{Discussion}

\section{ESCRT-III is expressed in developing barley endosperm}

ESCRT in plants is traditionally studied in Arabidopsis, conserved and distinct organization to fungi and metazoan cells is being established (Korbei et al. 2013; Reyes et al. 2011a) and recently, an assay for screening ESCRT target candidates has been proposed (Cai et al. 2014). There are two lines of evidence favouring the transfer of ESCRT knowledge to cereals and especially to cereal endosperm (1) the sall mutant in maize with its developmental phenotype of an abnormal high number of aleurone layers (Shen et al. 2003) and (2) observations in seeds that implicate the MVB in trafficking of SSPs (and possibly proteases) to the PSV (Reyes et al. 2011b, reviewed in Ibl and Stoger 2012). Here, a comparative basic inventory of evidently transcribed ESCRT-III subunits in barley, wheat, maize and rice has been compiled by searching public databases. Expression of ESCRT components in endosperm tissues in Arabidopsis and barley is covered by microarray data (Richardson and Mullen 2011; Sreenivasulu et al. 2008) and in maize by mass spectrometry (Walley et al. 2013).
In barley, we confirm by RT-PCR that all barley members in the annotated list are expressed in developing endosperm, including the paralogs of ESCRT-III members which are not present on the Affymetrix Barley1 GeneChip (HvVPS2.2, HvVPS2.3, HvSNF7c).

\section{Recombinant expression studies of ESCRT-III proteins}

Localization studies of ESCRT components are rare in seeds and even more so in cereal endosperm. Static images of fixed samples by confocal and electron microscopy have been provided for SAL1 in maize endosperm (Tian et al. 2007), but to our knowledge, no in vivo studies are present due to the technical complexity of cereal endosperm. Here, we present in vivo localization studies of HvSNF7a, HvVPS24 and HvVPS60a in different layers of developing barley endosperm.

Within this work, we constructed and expressed ESCRT-III components $\mathrm{C}$-terminally tagged with monomeric fluorophores (Shaner et al. 2004; Wiedenmann et al. 2004), i.e. versions with abolished intermolecular fluorophor interaction, and driven by the rice actin 1 promoter (McElroy et al. 1991). Aleurone and subaleurone cells were transiently transformed by particle bombardment. C-terminally tagged ESCRT-III fusion proteins have been used to elucidate, for example, their membrane binding behaviour in mammalian cells or ESCRT-III complex assembly in yeast, and Cterminally tagged AtVPS2.2 has been shown to partially complement a knock-out mutant in Arabidopsis (Hanson et al. 2008; Ibl et al. 2012; Teis et al. 2008). ESCRT-III, in contrast to ESCRT-I and II, is not a stable complex. Its monomeric components are recruited to form complexes at membranes with particular stoichiometry, and during or after membrane scission, they again dissociate fuelled by the action of the AAA+ ATPase VPS4 (Henne et al. 2013). C-terminally tagged ESCRT-III components exhibiting dominant negative behaviour have been shown (Ibl et al. 2012; Katsiarimpa et al. 2014; Pawliczek and Crump 2009; Strack et al. 2003; Teis et al. 2008), and this is explained by impaired autoinhibition to retain monomeric state. In our experimental setup, therefore, an enhanced accumulation (in comparison to endogenous levels) of ESCRT-III proteins at target membranes in the remodelling endomembrane system of barely endosperm might be attributed to both, distortion of ESCRTIII component stoichiometry by superimposed expression and by dominant negative effects due to C-terminal tagging. Furthermore, impaired dissociation of ESCRT-III complexes might lead to ectopic localization at and thus tagging of membranes further downstream in ESCRT pathways in which they are involved. Dominant negative effects in turn can be exploited to functionally test involvement of ESCRT-III components in protein sorting pathways in both cell layers. 

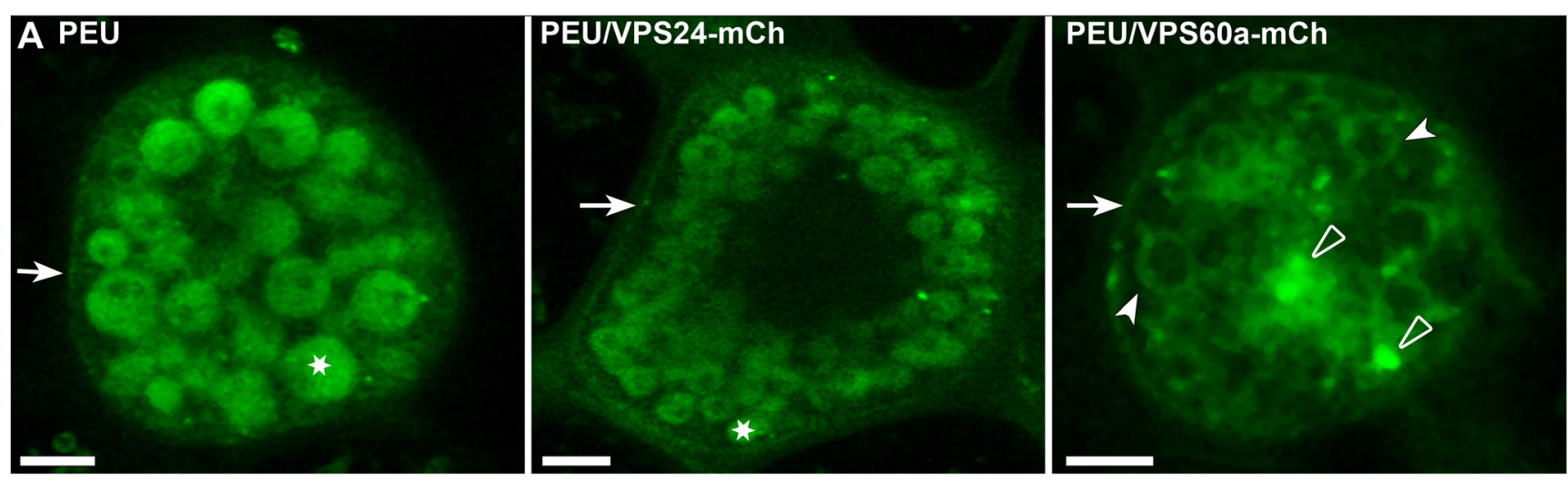

aleurone; $12 \mathrm{~h}$
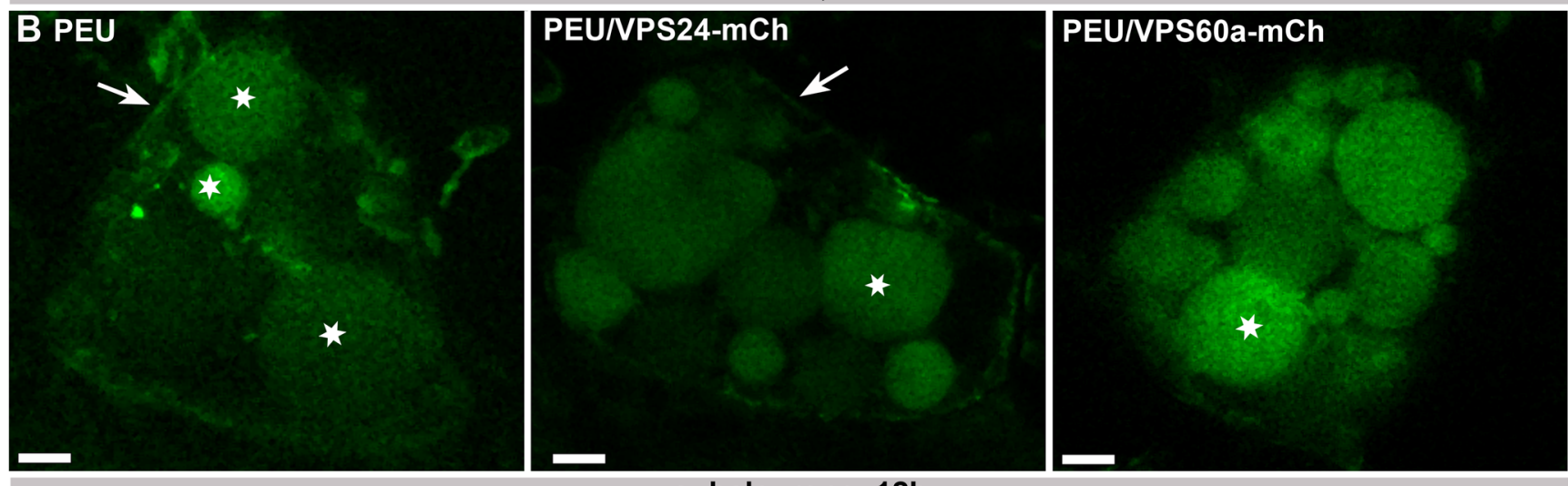

\section{subaleurone; $12 \mathrm{~h}$}
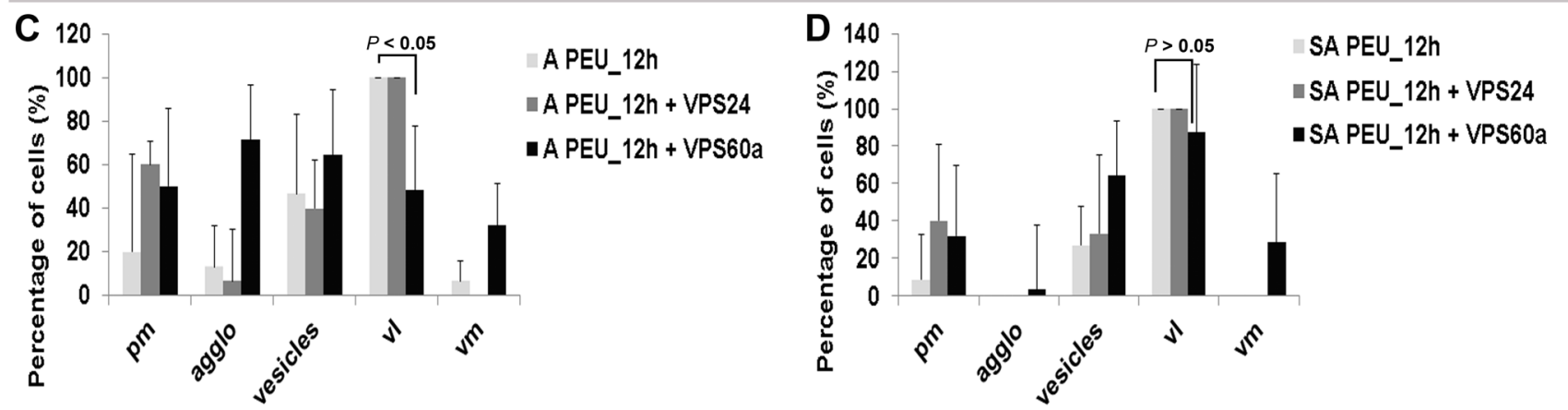

PEU localization in co-bombarded aleurone cells

PEU localization in co-bombarded subaleurone cells

\section{E 140}

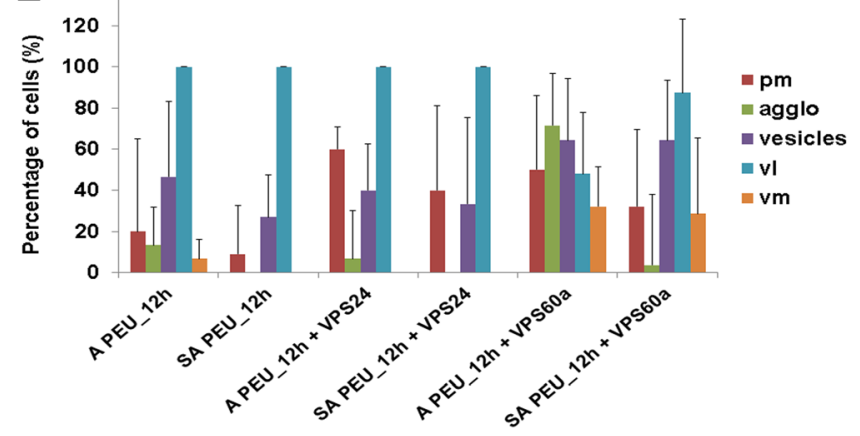

summary of PEU localization 
4 Fig. 6 Localization of PE and PEU in co-transformed aleurone and subaleurone cells. a PEU was sorted in the vacuole (asterisk) in aleurone cells. PEU is located at the PM (arrow) and sorted in vacuoles (asterisk) in co-bombarded HvVPS24-mCh cells. Note the affected sorting of PEU in co-transformed HvVPS60a-mCh cells, where PEU is localized at the pm (arrow), at agglomerations (open arrowheads) and at vacuolar membranes (arrowheads). b PEU is localized at the pm (arrowhead) and sorted in the vacuole in subaleurone cells (asterisks). In HvVPS24-mCh co-bombarded cells, PEU is localized weakly at the pm (arrowhead) and sorted in the vacuole. PEU is also sorted in the vacuole in co-transformed HvVPS60a-mCh cells (asterisk). Scale= $5 \mu \mathrm{m}$. c Statistical analyses of localizations of PEU in co-transformed HvVPS24-mCh and HvVPS60a-mCh aleurone cells. Biological replicates: 3 and 5 times for co-transformation with HvVPS24m-Ch and HvVPS60a-mCh, respectively; $n$ (co-transformed cells with HvVPS24$\mathrm{mCh} / \mathrm{HvVPS} 60 \mathrm{a}-\mathrm{mCh}$ : 15/28). d Statistical analyses of localization of PEU in co-transformed HvVPS24-mCh and HvVPS60a-mCh aleurone cells. Biological replicates: 3 and 6 times for co-transformation with HvVPS24m-Ch and HvVPS60a-mCh, respectively; $n$ (co-transformed cells with HvVPS24-mCh/HvVPS60a-mCh: 15/28). The difference in the localization of PEU in aleurone cells in the vacuolar lumen alone or in the presence of HvVPS60a-mCh is statistically significant $(P<0.05)$, whereas in subaleurone, the difference in the localization of PEU alone or in presence of HvVPS60a-mCh is not significant $(P>0.05)$. Plus ends of the standard deviation are indicated. e Summary of the statistical analyses of PEU in co-transformed HvVPS24m-Ch and HvVPS60a-mCh aleurone and subaleurone cells. Note the different localization of PEU in cotransformed HvVPS60a-mCh aleurone cells. Plus ends of the standard deviation are indicated. $A$ aleurone, $S A$ subaleurone, $p m$ plasma membrane, agglo agglomeration, $v l$ vacuolar lumen, $v m$ vacuolar membrane.

\section{HvSNF7a and HvVPS24 induces large agglomerations in barley endosperm}

Within this work, transiently overexpressed HvSNF7amEosFP localized at considerable agglomerations in barley endosperm. Recent Arabidopsis ESCRT protein interactome studies pointed out that Snf7A and Snf7B proteins are also capable of homotypic and heterotypic interactions, consistent with a conserved role for Snf7 oligomerization during ESCRT-III assembly in plants (Richardson and Mullen 2011; Shahriari et al. 2011). Richardson and Mullen (2011) further showed that the Arabidopsis Snf7A (i.e. HA-Snf7A) localized in BY-2 cells to punctate and large, globular like structures (Richardson and Mullen 2011). It was pointed out that these large globular structures may be also the result of the assembly of abnormal Snf7A oligomers as they were observed before in yeast and mammalian cells, respectively, to form large, higher-ordered polymers on the endosomal surface (Teis et al. 2010) and induce the formation of aberrant MVB-related structures upon their overexpression in cells (Peck et al. 2004; Boysen and Mitchell 2006). Thus, the large agglomerations induced by transiently expressed HvSNF7a$\mathrm{mEosFP}$ point to a protein with intact membrane association capacity and they indicate a functional role of HvSNF7a in barley endosperm.

Similarly, overexpressed Vps24 was reported to become membrane-associated and to perturb endosomal structure in mammalian cells (Lin et al. 2005). In barley endosperm, transient overexpression of HvVPS24-mEosFP induced prominent agglomerations in addition to cytosolic localization even though these compartments were smaller compared to HvSNF7a-mEos FP-induced compartments. These results support the hypothesis that the relative stoichiometry of ESCRT-III subunits is an important factor in ESCRT-III assembly (Teis et al. 2008) and that the increased steady-state association of ESCRT-III proteins with membranes caused by overexpression is inducing agglomerations of different sizes, causing dysfunction in the MVB pathway (Lin et al. 2005).

\section{Particular localization of HvVPS60a at the plasma membrane in barley aleurone}

Here, we present subcellular localization data of HvVPS60a in plants. Our microscopic analysis of transiently expressed HvVPS60a-mCh shows cytosolic localization together with a strong signal at small aggregates $(\sim 1 \mu \mathrm{m})$ and at the PM in barley aleurone.

Localization of ESCRT proteins at the PM has been reported in mammalian cells and plants (Ibl et al. 2012; Jimenez et al. 2014; Richardson et al. 2011). While the functional significance of the plasma membrane localization of ESCRT proteins is still unknown and needs to be established in planta, ESCRT components have been described in mammalian cells to be involved in plasma membrane repair (Jimenez et al. 2014) and in exosome secretion and composition (Colombo et al. 2013). As no single transformed HvVPS24 and HvSNF7a plasma membrane localization could be observed within this work in aleurone, we exclude that wounding by particle bombardment is responsible for the localization of HvVPS60a at the plasma membrane. Thus, we do not assume a specific role of HvVPS60a in plasma membrane repair. On the other hand, ESCRT-mediated secretion in plant cells has been discussed (Ibl et al. 2012), even though conclusive evidence is still missing. As it is known that membrane proteins dedicated for either lysosomal/vacuolar functions or exosomal release are sequestered into MVB intraluminal vesicles (ILV) (Katzmann et al. 2002; Piper and Katzmann 2007), we hypothesize that HvVPS60a is localized at MVBs that are finally fusing with the plasma membrane. As the recombinant HvVPS60a is possibly changing the steady-state assembly/ disassembly of ESCRT-III, HvVPS60a may remain at the MVB and thus at the plasma membrane after fusion. It is worth to mention that HvVPS24 could only be detected in the aleurone at the plasma membrane in $20 \%$ of cells compared to $80 \%$ of cells with HvVPS60a, indicating that HvVPS60a is possibly additionally involved in a different MVB trafficking pathway. This hypothesis is confirmed by recent data where SAL1 could be detected to a subfraction of MVBs in Arabidopsis embryo cells (Tian et al. 2007). Interestingly, biochemical and morphological evidence has been 
provided that the distinct fates (endocytosis and exocytosis) rely on distinct populations of MVBs that coexist within the same mammalian cell (Buschow et al. 2009; Mobius et al. 2002; White et al. 2006; Wubbolts et al. 2003). MVBs for both protein delivery to PSVs and involved in secretion were described in maize endosperm (Costa et al. 2003; Reyes et al. 2011b) but to our knowledge, no data have been provided in plants of morphologically identical but biochemically different MVBs. Thus, co-localization studies with a MVB marker (e.g. FYVE marker) and HvVPS60a and inhibitory treatments (e.g. wortmannin) will offer information about diverse MVBs within the aleurone.

\section{Spatially regulated localization of HvVPS60a in barley endosperm}

Transient transformation of HvVPS60a in transgenic barley OsTIP3::OsTIP3-GFP lines allowed us, first, to identify viable cells and, second, to differentiate aleurone from subaleurone cells. Previous work has shown that morphological changes of PSVs are cell layer dependent and particularly dynamic in subaleurone and starchy endosperm cells, consistent with a rapidly remodelling endomembrane system during development (Ibl et al. 2014). Our data point to a spatially regulated localization of HvVPS60a. As described, in aleurone, HvVPS60a is localized in the cytosol, at small agglomerations and at the plasma membrane, whereas in subaleurone, HvVPS60a is associated with PSV membranes.

Different localization of HvVPS60a in aleurone and subaleurone may reflect its varying function in protein sorting. In this study, we showed that the artificial MVB cargo PMAEGFP-UB (PEU) is endocytosed and delivered into the vacuole in barley endosperm. Since overexpressed HvVPS60a is associated with PSV membranes in subaleurone, we hypothesized that in subaleurone cells, a defective steady-state membrane association of HvVPS60a is induced by the different remodelling endomembrane system and possibly affecting protein targeting to the vacuole (Ibl et al. 2014). Recently, mis-localization of dominant negative ESCRT-III mutants to the tonoplast was described suggesting that the mutants were defective in membrane dissociation resulting in enhanced membrane association (Cai et al. 2014).

The necessity of the ESCRT-III steady-state membrane association is supported by the induced re-localization of HvVPS24 to the plasma membrane in aleurone and subaleurone, indicating that both proteins are acting in the same pathway. Surprisingly, co-bombardment studies of HvVPS60a and HvVPS24 with PEU in aleurone and subaleurone showed that in the presence of HvVPS60a, PEU is inefficiently targeted to the vacuolar lumen in aleurone. Contrary, PEU could be detected in a higher percentage of cells in the vacuolar lumen in the subaleurone in the presence of HvVPS60a. Thus, we conclude that
HvVPS60a has an inhibitory effect on targeting the monoubiquitinated plasma membrane protein in aleurone. In conclusion, our findings reinforce the concept of Richardson and Mullen (2011) that highlighted the very dynamic expression of Arabidopsis ESCRT across a wide range of organs, tissues and treatments possibly resulting in a complex interplay between the spatial and temporal regulation of the ESCRT machinery in Arabidopsis. Our observations suggest that the localization of HvVPS60a is spatially regulated in barley endosperm. Moreover, we show involvement of HvVPS60a in protein targeting of ubiquitinated protein towards the vacuole in aleurone. Our interpretation implies that the function of ESCRT-III depends on the cell layer in barley endosperm. These observations point out the necessity to study the ESCRT-III machinery at the molecular as well as at the cellular level in every single cell tissue, and it will be worth to include the temporal influence (developing stage) in the future studies to understand the spatial-temporal regulation of ESCRT-III in cereal endosperm. Transgenic ESCRT-III barley marker lines and ESCRT-III RNAi barley lines will pave the way to investigate the cell layer-specific steady-state assembly/disassembly of ESCRT-III and the function of ESCRT-III in barley endosperm.

Acknowledgments We are grateful to Yasushi Kawagoe for kindly sharing material and Swen Schellmann (University of Cologne) for the PMA-GFP and PMA-GFP-UB constructs. We thank the BOKU-VIBT Imaging Center for access and expertise. The authors would like to acknowledge financial support by the Austrian Science Fund FWF (P25736-B20 and I1461-B16).

Conflict of interest The authors do not have any conflict of interest.

Open Access This article is distributed under the terms of the Creative Commons Attribution License which permits any use, distribution, and reproduction in any medium, provided the original author(s) and the source are credited.

\section{References}

Babst M, Katzmann DJ, Estepa-Sabal EJ, Meerloo T, Emr SD (2002a) Escrt-III: an endosome-associated heterooligomeric protein complex required for mvb sorting. Dev Cell 3:271-282

Babst M, Katzmann DJ, Snyder WB, Wendland B, Emr SD (2002b) Endosome-associated complex, ESCRT-II, recruits transport machinery for protein sorting at the multivesicular body. Dev Cell 3: 283-289

Boothe J et al (2010) Seed-based expression systems for plant molecular farming. Plant Biotechnol J 8:588-606. doi:10.1111/j.1467-7652. 2010.00511.x

Boura E, Ivanov V, Carlson LA, Mizuuchi K, Hurley JH (2012) Endosomal sorting complex required for transport (ESCRT) complexes induce phase-separated microdomains in supported lipid bilayers. J Biol Chem 287:28144-28151. doi:10.1074/jbc.M112. 378646 
Boysen JH, Mitchell AP (2006) Control of Bro1-domain protein Rim20 localization by external $\mathrm{pH}$, ESCRT machinery, and the Saccharomyces cerevisiae Rim101 pathway. Mol Biol Cell 17(3): 1344-1353

Buchkovich NJ, Henne WM, Tang S, Emr SD (2013) Essential Nterminal insertion motif anchors the ESCRT-III filament during MVB vesicle formation. Dev Cell 27:201-214. doi:10.1016/j. devcel.2013.09.009

Buschow SI et al (2009) MHC II in dendritic cells is targeted to lysosomes or T cell-induced exosomes via distinct multivesicular body pathways. Traffic 10:1528-1542. doi:10.1111/j.1600-0854.2009. 00963.x

Caballe A, Martin-Serrano J (2011) ESCRT machinery and cytokinesis: the road to daughter cell separation. Traffic 12:1318-1326. doi:10. 1111/j.1600-0854.2011.01244.x

Cai Y, Zhuang X, Gao C, Wang X, Jiang L (2014) The arabidopsis endosomal sorting complex required for transport III regulates internal vesicle formation of the prevacuolar compartment and is required for plant development. Plant Physiol 165:1328-1343. doi: 10.1104/pp. 114.238378

Cannon EK et al (2011) POPcorn: an online resource providing access to distributed and diverse maize project data. Int J Plant Genomics 2011:923035. doi:10.1155/2011/923035

Carlton JG, Martin-Serrano J (2009) The ESCRT machinery: new functions in viral and cellular biology. Biochem Soc Trans 37:195-199. doi:10.1042/BST0370195

Colombo M et al (2013) Analysis of ESCRT functions in exosome biogenesis, composition and secretion highlights the heterogeneity of extracellular vesicles. J Cell Sci 126:5553-5565. doi:10.1242/jcs. 128868

Costa LM, Gutierrez-Marcos JF, Brutnell TP, Greenland AJ, Dickinson HG (2003) The globby1-1 (glo1-1) mutation disrupts nuclear and cell division in the developing maize seed causing alterations in endosperm cell fate and tissue differentiation. Development 130: 5009-5017. doi:10.1242/dev.00692

Ettema TJ, Bernander R (2009) Cell division and the ESCRT complex: a surprise from the archaea. Commun Integr Biol 2:86-88

Galili G (2004) ER-derived compartments are formed by highly regulated processes and have special functions in plants. Plant Physiol 136: 3411-3413. doi:10.1104/pp. 104.900125

Hanson PI, Cashikar A (2012) Multivesicular body morphogenesis. Annu Rev Cell Dev Biol 28:337-362. doi:10.1146/annurevcellbio-092910-154152

Hanson PI, Roth R, Lin Y, Heuser JE (2008) Plasma membrane deformation by circular arrays of ESCRT-III protein filaments. J Cell Biol 180:389-402. doi:10.1083/jcb.200707031

Henne WM, Buchkovich NJ, Zhao Y, Emr SD (2012) The endosomal sorting complex ESCRT-II mediates the assembly and architecture of ESCRT-III helices. Cell 151:356-371. doi:10.1016/j.cell.2012.08.039

Henne WM, Stenmark H, Emr SD (2013) Molecular mechanisms of the membrane sculpting ESCRT pathway. Cold Spring Harb Perspect Biol 5 doi:10.1101/cshperspect.a016766

Herberth S, Shahriari M, Bruderek M, Hessner F, Muller B, Hulskamp M, Schellmann S (2012) Artificial ubiquitylation is sufficient for sorting of a plasma membrane ATPase to the vacuolar lumen of Arabidopsis cells. Planta 236:63-77. doi:10.1007/s00425-012-1587-0

Hoh B, Hinz G, Jeong BK, Robinson DG (1995) Protein storage vacuoles form de novo during pea cotyledon development. J Cell Sci 108(Pt 1):299-310

Howard TL, Stauffer DR, Degnin CR, Hollenberg SM (2001) CHMP1 functions as a member of a newly defined family of vesicle trafficking proteins. J Cell Sci 114:2395-2404

Hurley JH, Emr SD (2006) The ESCRT complexes: structure and mechanism of a membrane-trafficking network. Annu Rev Biophys Biomol Struct 35:277-298. doi:10.1146/annurev.biophys.35. 040405.102126
Hurley JH, Hanson PI (2010) Membrane budding and scission by the ESCRT machinery: it's all in the neck. Nat Rev Mol Cell Biol 11: 556-566. doi:10.1038/nrm2937

Ibl V, Csaszar E, Schlager N, Neubert S, Spitzer C, Hauser MT (2012) Interactome of the plant-specific ESCRT-III component AtVPS2.2 in Arabidopsis thaliana. J Proteome Res 11:397-411. doi:10.1021/ pr200845n

Ibl V, Kapusi E, Arcalis E, Kawagoe Y, Stoger E (2014) Fusion, rupture, and degeneration: the fate of in vivo-labelled PSVs in developing barley endosperm. J Exp Bot. doi:10.1093/jxb/eru175

Ibl V, Stoger E (2012) The formation, function and fate of protein storage compartments in seeds. Protoplasma 249:379-392. doi:10.1007/ s00709-011-0288-Z

Jimenez AJ, Maiuri P, Lafaurie-Janvore J, Divoux S, Piel M, Perez F (2014) ESCRT machinery is required for plasma membrane repair. Science 343:1247136. doi:10.1126/science.1247136

Kapusi E, Hensel G, Coronado MJ, Broeders S, Marthe C, Otto I, Kumlehn J (2013) The elimination of a selectable marker gene in the doubled haploid progeny of co-transformed barley plants. Plant Mol Biol 81:149-160. doi:10.1007/s11103-012-9988-9

Katsiarimpa A, Anzenberger F, Schlager N, Neubert S, Hauser MT, Schwechheimer C, Isono E (2011) The arabidopsis deubiquitinating enzyme AMSH3 interacts with ESCRT-III subunits and regulates their localization. Plant Cell 23:3026-3040. doi:10.1105/tpc.111. 087254

Katsiarimpa A et al (2013) The deubiquitinating enzyme AMSH1 and the ESCRT-III subunit VPS2.1 are required for autophagic degradation in Arabidopsis. Plant Cell 25:2236-2252. doi:10.1105/tpc.113. 113399

Katsiarimpa A, Munoz A, Kalinowska K, Uemura T, Rojo E, Isono E (2014) The ESCRT-III-interacting deubiquitinating enzyme AMSH3 is essential for degradation of ubiquitinated membrane proteins in Arabidopsis thaliana. Plant Cell Physiol 55:727-736. doi: 10.1093/pcp/pcu019

Katzmann DJ, Babst M, Emr SD (2001) Ubiquitin-dependent sorting into the multivesicular body pathway requires the function of a conserved endosomal protein sorting complex, ESCRT-I. Cell 106: $145-155$

Katzmann DJ, Odorizzi G, Emr SD (2002) Receptor downregulation and multivesicular-body sorting. Nat Rev Mol Cell Biol 3:893-905. doi: $10.1038 / \mathrm{nrm} 973$

Korbei B, Moulinier-Anzola J, De-Araujo L, Lucyshyn D, Retzer K, Khan MA, Luschnig C (2013) Arabidopsis TOL proteins act as gatekeepers for vacuolar sorting of PIN2 plasma membrane protein. Curr Biol 23:2500-2505. doi:10.1016/j.cub.2013.10.036

Leung KF, Dacks JB, Field MC (2008) Evolution of the multivesicular body ESCRT machinery; retention across the eukaryotic lineage. Traffic 9:1698-1716. doi:10.1111/j.1600-0854.2008.00797.x

Li Z, Trick HN (2005) Rapid method for high-quality RNA isolation from seed endosperm containing high levels of starch. BioTechniques 38 : $872,874,876$

Lin Y, Kimpler LA, Naismith TV, Lauer JM, Hanson PI (2005) Interaction of the mammalian endosomal sorting complex required for transport (ESCRT) III protein hSnf7-1 with itself, membranes, and the AAA+ ATPase SKD1. J Biol Chem 280:12799-12809. doi: 10.1074/jbc.M413968200

Lindas AC, Karlsson EA, Lindgren MT, Ettema TJ, Bernander R (2008) A unique cell division machinery in the Archaea. Proc Natl Acad Sci U S A 105:18942-18946. doi:10.1073/pnas.0809467105

Magnusdottir A, Vidarsson H, Bjornsson JM, Orvar BL (2013) Barley grains for the production of endotoxin-free growth factors. Trends Biotechnol 31:572-580. doi:10.1016/j.tibtech.2013.06.002

McElroy D, Blowers AD, Jenes B, Wu R (1991) Construction of expression vectors based on the rice actin 1 (Act1) $5^{\prime}$ region for use in monocot transformation. Mol Gen Genet: MGG 231:150-160 
Mobius W et al (2002) Immunoelectron microscopic localization of cholesterol using biotinylated and non-cytolytic perfringolysin $\mathrm{O}$. J Histochem Cytochem: Off J Histochem Soc 50:43-55

Mornkham T, Wangsomnuk P, Fu Y-B, Wangsomnuk P, Jogloy S, Patanothai A (2013) Extractions of high quality RNA from the seeds of Jerusalem Artichoke and other plant species with high levels of starch and lipid. Plants 2:302-316

Moulinier-Anzola J, De-Araujo L, Korbei B (2014) Expression of Arabidopsis TOL genes. Plant Signal Behav 9:e28667

Olsen OA (2004) Nuclear endosperm development in cereals and Arabidopsis thaliana. The Plant Cell 16 (Suppl 1):S214-S227

Olsen LT, Divon HH, Al R, Fosnes K, Lid SE, Opsahl-Sorteberg HG (2008) The defective seed5 (des5) mutant: effects on barley seed development and HvDek1, HvCr4, and HvSal1 gene regulation. J Exp Bot 59:3753-3765. doi:10.1093/jxb/ern228

Onda Y, Kumamaru T, Kawagoe Y (2009) ER membrane-localized oxidoreductase Ero1 is required for disulfide bond formation in the rice endosperm. Proc Natl Acad Sci U S A 106:14156-14161. doi:10. 1073/pnas.0904429106

Pawliczek T, Crump CM (2009) Herpes simplex virus type 1 production requires a functional ESCRT-III complex but is independent of TSG101 and ALIX expression. J Virol 83:11254-11264. doi:10. 1128/jvi. 00574-09

Peck JW, Bowden ET, Burbelo PD (2004) Structure and function of human Vps20 and Snf7 proteins. Biochem J 377(Pt 3):693-700

Peel S, Macheboeuf P, Martinelli N, Weissenhorn W (2011) Divergent pathways lead to ESCRT-III-catalyzed membrane fission. Trends Biochem Sci 36:199-210. doi:10.1016/j.tibs.2010.09.004

Piper RC, Katzmann DJ (2007) Biogenesis and function of multivesicular bodies. Annu Rev Cell Dev Biol 23:519-547. doi:10.1146/annurev. cellbio.23.090506.123319

Rademacher T, Arcalis E, Stoger E (2009) Production and localization of recombinant pharmaceuticals in transgenic seeds. Methods Mol Biol 483:69-87. doi:10.1007/978-1-59745-407-0 5

Reyes FC, Buono R, Otegui MS (2011a) Plant endosomal trafficking pathways. Curr Opin Plant Biol 14:666-673. doi:10.1016/j.pbi. 2011.07.009

Reyes FC, Buono RA, Roschzttardtz H, Di Rubbo S, Yeun LH, Russinova E, Otegui MS (2014) A novel endosomal sorting complex required for transport (ESCRT) component in Arabidopsis thaliana controls cell expansion and development. J Biol Chem 289:4980-4988. doi:10.1074/jbc.M113.529685

Reyes FC, Chung T, Holding D, Jung R, Vierstra R, Otegui MS (2011b) Delivery of prolamins to the protein storage vacuole in maize aleurone cells. Plant Cell 23:769-784. doi:10.1105/tpc.110.082156

Richardson LG, Howard AS, Khuu N, Gidda SK, McCartney A, Morphy BJ, Mullen RT (2011) Protein-protein interaction network and subcellular localization of the Arabidopsis thaliana ESCRT machinery. Front Plant Sci 2:20. doi:10.3389/fpls.2011.00020

Richardson LG, Mullen RT (2011) Meta-analysis of the expression profiles of the Arabidopsis ESCRT machinery. Plant Signal Behav 6: $1897-1903$

Roxrud I, Stenmark H, Malerod L (2010) ESCRT Co Biol Cell 102:293318. doi:10.1042/BC20090161

Rusten TE, Stenmark H (2009) How do ESCRT proteins control autophagy? J Cell Sci 122:2179-2183. doi:10.1242/jcs.050021

Saisho D, Takeda K (2011) Barley: emergence as a new research material of crop science. Plant Cell Physiol 52:724-727. doi:10.1093/pcp/ pcr049

Samson RY, Bell SD (2009) Ancient ESCRTs and the evolution of binary fission. Trends Microbiol 17:507-513. doi:10.1016/j.tim.2009.08.003

Sanford JC, Smith FD, Russell JA (1993) Optimizing the biolistic process for different biological applications. Methods Enzymol 217:483509
Scheuring D et al (2012) Ubiquitin initiates sorting of Golgi and plasma membrane proteins into the vacuolar degradation pathway. BMC Plant Biol 12:164. doi:10.1186/1471-2229-12-164

Shahriari M, Richter K, Keshavaiah C, Sabovljevic A, Huelskamp M, Schellmann S (2011) The Arabidopsis ESCRT protein-protein interaction network. Plant Mol Biol 76:85-96. doi:10.1007/s11103-0119770-4

Shaner NC, Campbell RE, Steinbach PA, Giepmans BN, Palmer AE, Tsien RY (2004) Improved monomeric red, orange and yellow fluorescent proteins derived from Discosoma sp. red fluorescent protein. Nat Biotechnol 22:1567-1572. doi:10.1038/nbt1037

Shen B, Li C, Min Z, Meeley RB, Tarczynski MC, Olsen OA (2003) sall determines the number of aleurone cell layers in maize endosperm and encodes a class E vacuolar sorting protein. Proc Natl Acad Sci U S A 100:6552-6557. doi:10.1073/pnas.0732023100

Shewry PR, Napier JA, Tatham AS (1995) Seed storage proteins: structures and biosynthesis. Plant Cell 7:945-956. doi:10.1105/tpc.7.7. 945

Spallek T, Beck M, Ben Khaled S, Salomon S, Bourdais G, Schellmann S, Robatzek S (2013) ESCRT-I mediates FLS2 endosomal sorting and plant immunity. PLoS Genet 9:e1004035. doi:10.1371/journal. pgen. 1004035

Spitzer C, Reyes FC, Buono R, Sliwinski MK, Haas TJ, Otegui MS (2009) The ESCRT-related CHMP1A and B proteins mediate multivesicular body sorting of auxin carriers in Arabidopsis and are required for plant development. Plant Cell 21:749-766. doi:10. $1105 /$ tpc. 108.064865

Spitzer C et al (2006) The Arabidopsis elch mutant reveals functions of an ESCRT component in cytokinesis. Development 133:4679-4689. doi:10.1242/dev.02654

Sreenivasulu $\mathrm{N}$ et al (2008) Barley grain maturation and germination: metabolic pathway and regulatory network commonalities and differences highlighted by new MapMan/PageMan profiling tools. Plant Physiol 146:1738-1758. doi:10.1104/pp. 107.111781

Stoger E, Ma JK, Fischer R, Christou P (2005) Sowing the seeds of success: pharmaceutical proteins from plants. Curr Opin Biotechnol 16:167-173. doi:10.1016/j.copbio.2005.01.005

Strack B, Calistri A, Craig S, Popova E, Gottlinger HG (2003) AIP1/ ALIX is a binding partner for HIV-1 p6 and EIAV p9 functioning in virus budding. Cell 114:689-699

Tamura K, Stecher G, Peterson D, Filipski A, Kumar S (2013) MEGA6: Molecular Evolutionary Genetics Analysis version 6.0. Mol Biol Evol 30:2725-2729. doi:10.1093/molbev/mst197

Teis D, Saksena S, Emr SD (2008) Ordered assembly of the ESCRT-III complex on endosomes is required to sequester cargo during MVB formation. Dev Cell 15:578-589. doi:10.1016/j.devcel.2008.08.013

Teis D, Saksena S, Judson BL, Emr SD (2010) ESCRT-II coordinates the assembly of ESCRT-III filaments for cargo sorting and multivesicular body vesicle formation. Embo J 29:871-883. doi: 10.1038/emboj.2009.408

Tian Q et al (2007) Subcellular localization and functional domain studies of DEFECTIVE KERNEL1 in maize and Arabidopsis suggest a model for aleurone cell fate specification involving CRINKLY4 and SUPERNUMERARY ALEURONE LAYER1. Plant Cell 19: 3127-3145. doi:10.1105/tpc.106.048868

Walley JW, Shen Z, Sartor R, Wu KJ, Osborn J, Smith LG, Briggs SP (2013) Reconstruction of protein networks from an atlas of maize seed proteotypes. Proc Natl Acad Sci U S A 110:E4808-E4817. doi: 10.1073/pnas.1319113110

Wang Y et al (2010) OsRab5a regulates endomembrane organization and storage protein trafficking in rice endosperm cells. Plant J: Cell Mol Biol 64:812-824. doi:10.1111/j.1365-313X.2010.04370.x

Webster BM, Colombi P, Jager J, Lusk CP (2014) Surveillance of nuclear pore complex assembly by ESCRT-III/Vps4. Cell 159:388-401. doi:10.1016/j.cell.2014.09.012 
White IJ, Bailey LM, Aghakhani MR, Moss SE, Futter CE (2006) EGF stimulates annexin 1-dependent inward vesiculation in a multivesicular endosome subpopulation. Embo J 25:1-12. doi:10. 1038/sj.emboj.7600759

Wiedenmann J et al (2004) EosFP, a fluorescent marker protein with UVinducible green-to-red fluorescence conversion. Proc Natl Acad Sci U S A 101:15905-15910. doi:10.1073/pnas.0403668101

Williams RL, Urbe S (2007) The emerging shape of the ESCRT machinery. Nat Rev Mol Cell Biol 8:355-368. doi:10.1038/nrm2162

Winter V, Hauser MT (2006) Exploring the ESCRTing machinery in eukaryotes. Trends Plant Sci 11:115-123. doi:10.1016/j.tplants. 2006.01.008

Wubbolts R et al (2003) Proteomic and biochemical analyses of human B cell-derived exosomes. Potential implications for their function and multivesicular body formation. J Biol Chem 278:10963-10972. doi: 10.1074/jbc.M207550200

Zamborlini A, Usami Y, Radoshitzky SR, Popova E, Palu G, Gottlinger H (2006) Release of autoinhibition converts ESCRT-III components into potent inhibitors of HIV-1 budding. Proc Natl Acad Sci U S A 103:19140-19145. doi:10.1073/pnas.0603788103

Zhang XQ, Hou P, Zhu HT, Li GD, Liu XG, Xie XM (2013) Knockout of the VPS22 component of the ESCRT-II complex in rice (Oryza sativa L.) causes chalky endosperm and early seedling lethality. Mol Biol Rep 40:3475-3481. doi:10.1007/ s11033-012-2422-1

Zheng Y, Wang Z (2014) Protein accumulation in aleurone cells, subaleurone cells and the center starch endosperm of cereals. Plant Cell Rep. doi:10.1007/s00299-014-1651-2 